\title{
Simple model of the slingshot effect
}

\author{
Gaetano Fiore ${ }^{1,3}$ and Sergio De Nicola ${ }^{2,3}$ \\ ${ }^{1}$ Dip. di Matematica e Applicazioni, Università di Napoli "Federico II", \\ Complesso Universitario M. S. Angelo, Via Cintia, 80126 Napoli, Italy \\ ${ }^{2}$ SPIN-CNR, Complesso MSA, Via Cintia, 80126 Napoli, Italy \\ ${ }^{3}$ INFN, Sez. di Napoli, Complesso MSA, Via Cintia, 80126 Napoli, Italy
}

(Received 13 April 2016; published 21 July 2016)

\begin{abstract}
We present a detailed quantitative description of the recently proposed "slingshot effect." Namely, we determine a broad range of conditions under which the impact of a very short and intense laser pulse normally onto a low-density plasma (or matter locally completely ionized into a plasma by the pulse) causes the expulsion of a bunch of surface electrons in the direction opposite to the one of propagation of the pulse, and the detailed, ready-for-experiments features of the expelled electrons (energy spectrum, collimation, etc). The effect is due to the combined actions of the ponderomotive force and the huge longitudinal field arising from charge separation. Our predictions are based on estimating $3 \mathrm{D}$ corrections to a simple, yet powerful plane 2-fluid magnetohydrodynamic (MHD) model where the equations to be solved are reduced to a system of Hamilton equations in one dimension (or a collection of) which become autonomous after the pulse has overcome the electrons. Experimental tests seem to be at hand. If confirmed by the latter, the effect would provide a new extraction and acceleration mechanism for electrons, alternative to traditional radio-frequency-based or Laser-Wake-Field ones.
\end{abstract}

DOI: 10.1103/PhysRevAccelBeams.19.071302

\section{INTRODUCTION AND SETUP}

Laser-driven plasma-based acceleration (LPA) mechanisms were first conceived by Tajima and Dawson in 1979 [1] and have been intensively studied since then. In particular, after the rapid development [2,3] of chirped pulse amplification laser technology-making available compact sources of intense, high-power, ultrashort laser pulses - the laser wake field acceleration (LWFA) mechanism [1,4,5] allows us to generate extremely high acceleration gradients $(>1 \mathrm{GV} / \mathrm{cm})$ by plasma waves involving huge charge density variations. Since 2004 experiments have shown that LWFA in the so-called bubble (or blowout) regime can produce electron bunches of high quality (i.e. very good collimation and small energy spread), energies of up to hundreds of $\mathrm{MeVs}$ [6-8] or more recently even GeVs $[9,10]$. This allows a revolution in acceleration techniques of charged particles, with a host of potential applications in research (particle physics, materials science, structural biology, etc.) as well as applications in medicine, optycs, etc.

In the LWFA and its variations the laser pulse traveling in the plasma leaves a wakefield of plasma waves behind; a bunch of electrons (either externally [11] or self-injected [12]) can be accelerated "surfing" one of these plasma

Published by the American Physical Society under the terms of the Creative Commons Attribution 3.0 License. Further distribution of this work must maintain attribution to the author(s) and the published article's title, journal citation, and DOI. waves and exit the plasma sample just behind the pulse, in the same direction of propagation of the latter (forward expulsion). In Ref. [13] a new LPA mechanism, named the slingshot effect, has been proposed, in which a bunch of electrons are expected to be accelerated and expelled backwards from a low-density plasma sample shortly after the impact of a suitable ultrashort and ultraintense laser pulse in the form of a pancake normally onto the plasma (see Fig. 1). The surface electrons (i.e. plasma electrons in a thin layer just beyond the vacuum-plasma interface) first are all displaced forward (with respect to the ions) by the ponderomotive force $F_{p}:=\left\langle-e\left(\frac{v}{c} \times \boldsymbol{B}\right)^{z}\right\rangle$ generated by the pulse, leaving a layer of ions completely depleted of electrons (here \langle\rangle is the average over a period of the laser carrier wave, $\boldsymbol{E}, \boldsymbol{B}$ are the electric and magnetic fields, $\boldsymbol{v}$ is the electron velocity, $c$ is the speed of light, $\hat{z}$ is the direction of propagation of the laser pulse); $F_{p}$ is positive (negative) while the modulating amplitude $\epsilon_{s}$ of the pulse respectively grows (decreases). These electrons are then pulled back by the longitudinal electric force $F_{e}^{z}=-e E^{z}$ exerted by the ions and the other electrons, and leave the plasma. (In the meantime, the pulse proceeds deeper into the plasma, generating a wakefield.) Tuning the electron density in the range where the plasma oscillation period $T_{H}$ [14] is about twice the pulse duration $\tau$, we can make these electrons invert their motion when they are reached by the maximum of $\epsilon_{s}$, so that the negative part of $F_{p}$ (due to the subsequent decrease of $\epsilon_{s}$ ) adds to $F_{e}^{z}$ in accelerating them backwards; thus the total work $W=\int_{0}^{\tau} d t F_{p}\left\langle v^{z}\right\rangle$ done by the ponderomotive force is maximal [15]. Provided the 


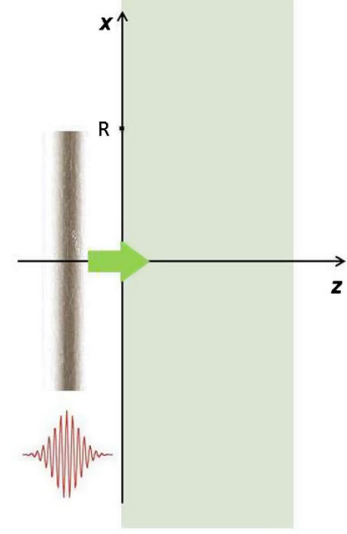

(a) Free transverse EM wave + plasma at rest (plane idealization)
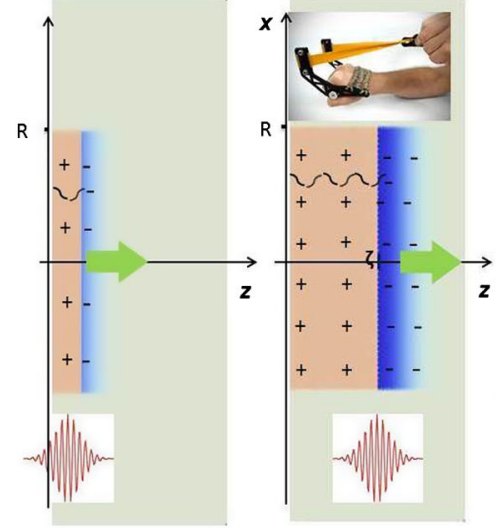

(b) Boost by the ponderomotive force exerted by the EM wave (plane wave idealization)

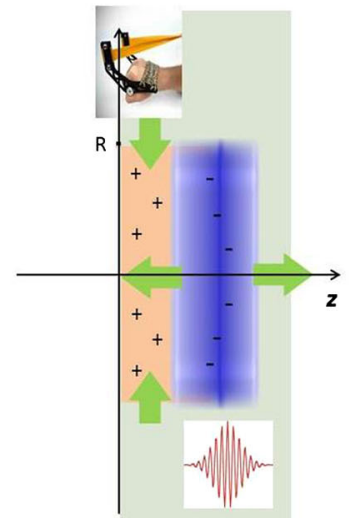

(c) The electric force due to the separation of charges boosts the electrons backwards: like a SLINGSHOT (plane wave idealization)

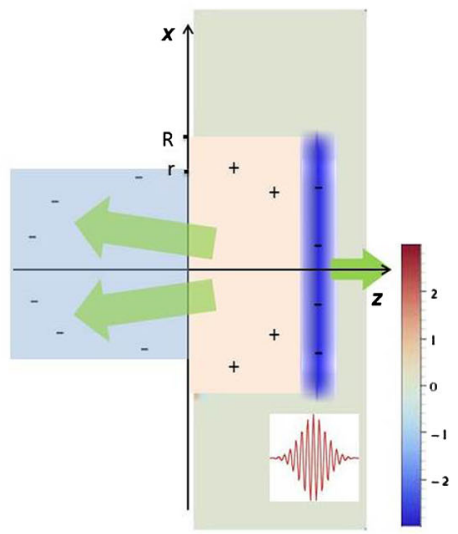

(d) Since $\mathrm{R}<\infty$, the Coulomb attraction by ions $\rightarrow 0$ as $z_{e} \rightarrow-\infty$, and allows $z_{e} \rightarrow-\infty$

FIG. 1. Schematic stages of the slingshot effect.

laser spot size $R$ is sufficiently small a significant part of the expelled electrons will have enough energy to win the attraction by ions and escape to infinity.

Very short $\tau$ 's and huge nonlinearities make approximation schemes based on Fourier analysis and related methods (slowly varying amplitude approximation, frequencydependent refractive indices,...) inconvenient. On the contrary, in the relevant space-time region a MHD description of the impact is self-consistent, simple and predictive (collisions are negligible, and recourse to kinetic theory is not needed). Here we develop and improve the 2-fluid MHD approach introduced in $[13,16]$ and apply it to determine a broad range of conditions enabling the effect, as well as detailed quantitative predictions about it (a brief summary is given in $[17,18])$. In section II we study the plane problem $(R=\infty)$ and show that for sufficiently low density and small times (after the impact) we can neglect the radiative corrections [backreaction of the plasma on the electromagnetic (EM) field (3)] and determine the motion of the surface electrons in the bulk by (numerically) solving a single system of two coupled first order ordinary differential equations of Hamiltonian form, if the initial density $\widetilde{n_{0}}$ is step-shaped, or a collection of such systems, otherwise; the role of "time" is played by the lightlike coordinate $\xi=c t-z$. The rough model of [13] considered only stepshaped $\widetilde{n_{0}}$ and was based on neglecting $F_{e}^{z}$ during the forward motion, $F_{p}$ during the backward motion of the electrons; the estimates could be considered reliable only for very low, unrealistic $\widetilde{n_{0}}$. Here $\widetilde{n_{0}}$ needs no longer to be so low, nor step-shaped, as in [13], because we take $F_{e}^{z}, F_{p}$ in due account during the whole motion of the electrons. In Sec. III we heuristically modify the potential energy outside the bulk to account for finite $R$ and determine a $R$-range such that the motion of the surface electrons coming from some inner cylinder $\rho^{2} \leq r^{2}<R^{2}$ be (by causality) well approximated by the solution of the correspondingly modified Hamilton equations; we then find which electrons indeed escape to infinity and estimate in detail their final energy spectrum, collimation, total number, charge and energy. To be specific, in Sec. IV we specialize predictions to potential experiments at the FLAME facility (LNF, Frascati) or the ILIL laboratory (INO-CNR, Pisa). We welcome 3D simulations and experiments checking these predictions; the experimental conditions are at hand in many laboratories. In Sec. V we discuss the results, the conditions for their validity and draw the conclusions.

As a context remark, we recall that relatively simple 2-fluid magnetohydrodynamic models can be used also to describe the complicated physics of the impact of very intense and short laser pulses on overdense solid targets. If the density gradient of the target is sufficiently steep, the massive displacement of electrons (induced by the ponderomotive force) with respect to ions (named snowplow in $[19,20])$ produces a longitudinal electric force which may accelerate also protons or other light ions, either backward or forward, by the so-called skin-layer ponderomotive acceleration [21] or relativistically induced transparency acceleration $[19,20]$ mechanisms.

\section{A. The 2-fluid magnetohydrodynamic framework}

The setup is as follows. We assume that the plasma is initially neutral, unmagnetized and at rest with electron (and proton) density equal to zero in the region $z<0$. We describe the plasma as consisting of a static background fluid of ions (the motion of ions can be neglected during the short time interval in which the effect occurs) and a fully relativistic collisionless fluid of electrons, with the "plasma + EM field" system fulfilling the Lorentz-Maxwell and the continuity equations. We show a posteriori that such a magnetohydrodynamic (MHD) treatment is self-consistent in the spacetime region of interest. We denote as $\boldsymbol{x}_{e}(t, \boldsymbol{X})$ the position at time $t$ of the electrons' fluid element initially located at $\boldsymbol{X} \equiv(X, Y, Z)$, and for each fixed $t$ as $\boldsymbol{X}_{e}(t, \boldsymbol{x})$ the inverse map $[\boldsymbol{x} \equiv(x, y, z)]$. For brevity, we refer to such a 
fluid element as to the " $\boldsymbol{X}$ electrons"; to the fluid elements with arbitrary $X, Y$ and specified $Z$, or with $X$ in a specified region $\Omega$, respectively as the " $Z$ electrons" or the " $\Omega$ electrons". We denote as $m, n_{e}, \boldsymbol{v}_{e}$ the electron mass, Eulerian density and velocity and often use the dimensionless fields $\quad \boldsymbol{\beta}_{e} \equiv \boldsymbol{v}_{e} / c, \quad \boldsymbol{u}_{e} \equiv \boldsymbol{p}_{e} / m c=\boldsymbol{\beta}_{e} / \sqrt{1-\boldsymbol{\beta}_{e}^{2}}$, $\gamma_{e} \equiv 1 / \sqrt{1-\boldsymbol{\beta}_{e}^{2}}=\sqrt{1+\boldsymbol{u}_{e}^{2}}$. The equations of motion are

$$
\begin{aligned}
\frac{d \boldsymbol{p}_{e}}{d t} & =-e\left(\boldsymbol{E}+\frac{\boldsymbol{v}_{e}}{c} \wedge \boldsymbol{B}\right), \\
\partial_{t} \boldsymbol{x}_{e}(t, \boldsymbol{X}) & =\boldsymbol{v}_{e}\left[t, \boldsymbol{x}_{e}(t, \boldsymbol{X})\right]
\end{aligned}
$$

in CGS units $\left(d / d t \equiv \partial_{t}+\boldsymbol{v}_{e} \cdot \nabla_{x}\right.$ is the electrons' material derivative) and the initial conditions are $\boldsymbol{p}_{e}(0, \boldsymbol{X})=0$, $\boldsymbol{x}_{e}(0, \boldsymbol{X})=\boldsymbol{X}$ for $Z \geq 0$. The Lagrangian fields depend on $t, \boldsymbol{X}$, rather than on $t, \boldsymbol{x}$, and are distinguished by a tilde, e.g. $\tilde{n}_{e}(t, \boldsymbol{X})=n_{e}\left[t, \boldsymbol{x}_{e}(t, \boldsymbol{X})\right]$. The continuity equation $d n_{e} / d t+$ $n_{e} \nabla_{x} \cdot v_{e}=0$ follows from the local conservation of the number of electrons, which amounts to

$$
\tilde{n}_{e}(t, \boldsymbol{X}) \operatorname{det}\left(\frac{\partial \boldsymbol{x}_{e}}{\partial \boldsymbol{X}}\right)=\widetilde{n_{0}}(\boldsymbol{X}) \equiv \tilde{n}_{e}(0, \boldsymbol{X})
$$

We assume that $\widetilde{n_{0}}$ is independent of $X, Y$ and, as said, vanishes if $Z<0$; also as a warm-up to more general $Z$-dependence, we start by studying the case that it is constant in the region $Z \geq 0: \widetilde{n_{0}}(Z)=n_{0} \theta(Z)$, where $\theta$ is the Heaviside step function. We consider a purely transverse EM pulse in the form of a pancake with cylindrical symmetry around the $z$-axis, propagating in the positive $\hat{z}$ direction and hitting the plasma surface $z=0$ at $t=0$. We schematize the pulse as a free plane pulse multiplied by a "cutoff" function $\chi_{R}(\rho)$ which is approximately equal to 1 for $\rho \equiv \sqrt{x^{2}+y^{2}} \leq R$ and rapidly goes to zero for $\rho>R$ (with some finite radius $R$, see Fig. 1(a))

$$
\boldsymbol{E}^{\perp}(t, \boldsymbol{x})=\boldsymbol{\epsilon}^{\perp}(c t-z) \chi_{R}(\rho), \quad \boldsymbol{B}^{\perp}=\hat{z} \times \boldsymbol{E}^{\perp}
$$

[in particular we consider $\chi_{R}(\rho) \equiv \theta(R-\rho)$ ]; the "pump" $\boldsymbol{\epsilon}^{\perp}(\xi)$ vanishes outside some finite interval $0<\xi<l$ [22].

\section{PLANE WAVE IDEALIZATION}

In the plane problem $(R=\infty)$ the invertibility of $\boldsymbol{x}_{e}: \boldsymbol{X} \mapsto \boldsymbol{x}$ for all fixed $t$ amounts to $z_{e}(t, Z)$ being strictly increasing with respect to $Z$ for all $t$. Equation (2) becomes

$$
\left[\tilde{n}_{e} \frac{\partial z_{e}}{\partial Z}\right](t, Z)=\widetilde{n_{0}}(Z) \Leftrightarrow n_{e}(t, z)=\widetilde{n_{0}}\left[Z_{e}(t, z)\right] \frac{\partial Z_{e}}{\partial z}(t, z) .
$$

Regarding ions as immobile, the Maxwell equations imply [16] that the longitudinal component of the electric field is related to $\tilde{N}(Z) \equiv \int_{0}^{Z} d Z^{\prime} \widetilde{n_{0}}\left(Z^{\prime}\right)$ (the number of electrons per unit surface in the layer $0 \leq Z^{\prime} \leq Z$ ) by

$$
E^{z}(t, z)=4 \pi e\left\{\tilde{N}(z)-\tilde{N}\left[Z_{e}(t, z)\right]\right\} .
$$

We partially fix the gauge [16] imposing that the transverse (with respect to $\hat{z}$ ) vector potential itself is independent of $x, y$, and hence is the physical observable $\boldsymbol{A}^{\perp}(t, z)=-\int_{-\infty}^{t} d t^{\prime} c \boldsymbol{E}^{\perp}\left(t^{\prime}, z\right)$; then $c \boldsymbol{E}^{\perp}=-\partial_{t} \boldsymbol{A}^{\perp}, \boldsymbol{B}=$ $\boldsymbol{B}^{\perp}=\hat{z} \wedge \partial_{z} \boldsymbol{A}^{\perp}$. As known, the transverse component of the Lorentz equation (1) implies $\boldsymbol{p}_{e}^{\perp}-\frac{e}{c} \boldsymbol{A}^{\perp}=$ const on the trajectory of each electron; this is zero at $t=0$, hence $\boldsymbol{p}_{e}^{\perp}=m c \boldsymbol{u}_{e}^{\perp}=e \boldsymbol{A}^{\perp} / c$. Hence $\boldsymbol{u}_{e}^{\perp}$ is determined in terms of $\boldsymbol{A}^{\perp}$. As in [16], we introduce the positive-definite field

$$
s_{e} \equiv \gamma_{e}-u_{e}^{z}
$$

which we name electron s-factor. $u_{e}^{z}, \gamma_{e}, \boldsymbol{\beta}_{e}^{\perp}, \beta_{e}^{z}$ are recovered from $\boldsymbol{u}_{e}^{\perp}, s_{e}$ through the formulas (44) of [16]:

$\gamma_{e}=\frac{1+\boldsymbol{u}_{e}^{\perp 2}+s_{e}^{2}}{2 s_{e}}, \quad u_{e}^{z}=\frac{1+\boldsymbol{u}_{e}^{\perp 2}-s_{e}^{2}}{2 s_{e}}, \quad \boldsymbol{\beta}_{e}=\frac{\boldsymbol{u}_{e}}{\gamma_{e}}$.

Remarkably, all of (7) are rational functions of $\boldsymbol{u}_{e}^{\perp}, s_{e}$ (no square roots appear). Moreover, fast oscillations of $\boldsymbol{u}_{e}^{\perp}$ affect $\gamma_{e}, u_{e}^{z}$ but not $s_{e}$ [see the comments after (15)]. For these reasons it is convenient to use $\boldsymbol{u}_{e}^{\perp}, s_{e}$ instead of $\boldsymbol{u}_{e}^{\perp}, u_{e}^{z}$ as independent unknowns. The evolution equation of $s_{e}$ (difference of the ones of $\gamma_{e}, u_{e}^{z}$; the former is the scalar product of $(1)_{1}$ with $\left.\boldsymbol{p}_{e} / \gamma_{e} m^{2} c^{2}\right)$ reads

$$
\gamma_{e} \frac{d s_{e}}{d t}=\frac{e E^{z}}{m c} s_{e}+\left(\partial_{t}+c \partial_{z}\right) \boldsymbol{u}_{e}^{\perp 2} .
$$

The Maxwell equation for $\boldsymbol{A}^{\perp}$ takes the form $\left(\partial_{0}^{2}-\partial_{z}^{2}\right) \boldsymbol{A}^{\perp}+\boldsymbol{A}^{\perp} 4 \pi e^{2} n_{e} / m c^{2} \gamma_{e}=0$; Eq. (3) with $R=\infty$ implies $\boldsymbol{A}^{\perp}(t, z)=\boldsymbol{\alpha}^{\perp}(c t-z)$ for $t \leq 0$, where $\boldsymbol{\alpha}^{\perp}(\xi) \equiv$ $-\int_{-\infty}^{\xi} d \xi^{\prime} \boldsymbol{\epsilon}^{\perp}\left(\xi^{\prime}\right)$. Using the Green function of the D'Alembertian $\partial_{0}^{2}-\partial_{z}^{2}$, abbreviating $x \equiv(t, z)$, these equations can be equivalently reformulated as the integral equation (42) of [16]

$$
\begin{aligned}
& \boldsymbol{A}^{\perp}(t, z)-\boldsymbol{\alpha}^{\perp}(c t-z)=-\int_{D_{x} \cap T} d t^{\prime} d z^{\prime}\left[\frac{2 \pi e^{2} n_{e}}{m c \gamma_{e}} \boldsymbol{A}^{\perp}\right]\left(x^{\prime}\right) \\
& D_{x} \equiv\left\{\left(t^{\prime}, z^{\prime}\right)\left|t^{\prime} \leq t,\right| z-z^{\prime} \mid \leq c t-c t^{\prime}\right\}, \quad T \equiv\{x|| z \mid<c t\} .
\end{aligned}
$$

The past, future causal cones $D_{x}, T$, the supports of $\boldsymbol{A}^{\perp}$, $\widetilde{n_{0}}(z)$, and their intersections are shown in Fig. 2. For $t<0$ $D_{x} \cap T$ is empty, and the right-hand side of $(9)_{1}$ is zero, as it must be. Below we shall analyze the consequences of neglecting it also for small $t$, and determine the range of validity of such an approximation. 


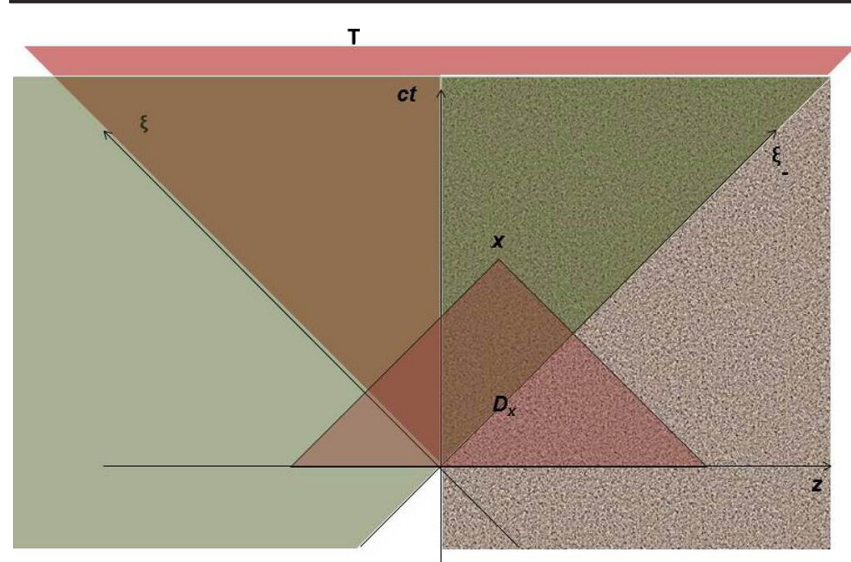

FIG. 2. Past (light brown) and future (purple) causal cones $D_{x}$, $T$; supports of $\boldsymbol{A}^{\perp}$ (light green) and $\widetilde{n_{0}}(z)$ (anthracite).

\section{A. Motion of the electrons}

Let $\quad \hat{\boldsymbol{u}}^{\perp}(\xi) \equiv e \alpha^{\perp}(\xi) / m c^{2}, \quad v(\xi) \equiv \hat{\boldsymbol{u}}^{\perp 2}(\xi)$,

$$
F_{e}^{z}(z, Z) \equiv-4 \pi e^{2}\{\tilde{N}(z)-\tilde{N}(Z)\}
$$

$\tilde{F}_{e}^{z}(t, Z) \equiv F_{e}^{z}\left[z_{e}(t, Z), Z\right]$ is the longitudinal electric force acting on the $Z$ electrons at time $t$; it is conservative, as it depends on $t$ only through $z_{e}(t, Z)$. The approximation $\boldsymbol{A}^{\perp}(t, z)=\boldsymbol{\alpha}^{\perp}(c t-z)$ implies $\boldsymbol{u}_{e}^{\perp}(t, z)=\hat{\boldsymbol{u}}^{\perp}(c t-z)$, and the last term of (8) vanishes. Replacing (5) in the Lagrangian version of (8), we find for each $Z \geq 0$ the equation $\tilde{\gamma}_{e} \partial_{t} \tilde{s}_{e}=-\tilde{s}_{e} \widetilde{F_{e}^{z}} / m c$. The initial condition is $\tilde{s}_{e}(0, Z) \equiv 1$. The other equation to be solved is $(1)_{2}$ with the initial condition $\boldsymbol{x}_{e}(0, \boldsymbol{X})=\boldsymbol{X}$. By (7) one is thus led to the Cauchy problems (parametrized by $Z \geq 0$ )

$$
\partial_{t} \frac{z_{e}-Z}{c}=\frac{1+v\left[c t-z_{e}(t, Z)\right]-\tilde{s}_{e}^{2}}{1+v\left[c t-z_{e}(t, Z)\right]+\tilde{s}_{e}^{2}}, \quad \partial_{t} \tilde{s}_{e}=\frac{-\tilde{s}_{e}}{\tilde{\gamma}_{e} m c} \widetilde{F_{e}^{z}}
$$

$$
z_{e}(0, Z)-Z=0, \quad \tilde{s}_{e}(0, Z)=1 .
$$

$\boldsymbol{x}_{e}^{\perp}(t, \boldsymbol{X})$ is obtained from the solutions of (11)-(12) using (1), (7):

$$
\boldsymbol{x}_{e}^{\perp}(t, \boldsymbol{X})=\boldsymbol{X}^{\perp}+\int_{0}^{t} d t^{\prime} c \boldsymbol{\beta}_{e}^{\perp}\left[t^{\prime}, z_{e}\left(t^{\prime}, Z\right)\right] .
$$

For all fixed $Z$ the map $t \mapsto \tilde{\xi}(t, Z) \equiv c t-z_{e}(t, Z)$ is invertible, because the speed of electrons is always smaller than $c$. We can simplify (11) by the change of variables $(t, Z) \mapsto(\xi, Z)$, making the argument of $v$ an independent variable. Denoting the dependence on $(\xi, Z)$ by a caret [e.g. $\left.\hat{s}(\xi, Z)=\tilde{s}_{e}(t, Z)\right]$ and introducing the displacement from the initial position $\hat{\Delta}(\xi, Z) \equiv \hat{z}_{e}(\xi, Z)-Z$, we find $\partial_{\xi}=\left(\tilde{\gamma}_{e} / c \tilde{s}_{e}\right) \partial_{t}$, and (11) becomes
$\hat{\Delta}^{\prime}=\frac{1+v}{2 \hat{s}^{2}}-\frac{1}{2}, \quad \hat{s}^{\prime}=\frac{4 \pi e^{2}}{m c^{2}}\{\tilde{N}[\hat{\Delta}+Z]-\tilde{N}(Z)\}$

(the prime means differentiation with respect to $\xi$ ). For $\xi \leq$ $0 v(\xi) \equiv 0, \hat{\Delta}, \hat{s}$ remain constant, and we can replace the initial conditions $\hat{\Delta}(-Z, Z)=0, \hat{s}(-Z, Z)=1$ by

$$
\hat{\Delta}(0, Z)=0, \quad \hat{s}(0, Z)=1 .
$$

An alternative derivation of (14)-(15) with a deeper insight on the role of the $s$-factor is given in [23]. In the zero density limit $\tilde{N}(Z) \equiv 0, \hat{s} \equiv 1$, (14)-(15) is integrable, and all unknowns are determined explicitly from $\left.\boldsymbol{\epsilon}^{\perp}[16,24]\right)$. As $v \geq 0$, even if $\boldsymbol{\epsilon}^{\perp}, \boldsymbol{u}^{\perp}, v$ oscillate fast with $\xi$, integrating (14) makes relative oscillations of $\hat{\Delta}$ much smaller than those of $v$ and those of $\hat{s}$ much smaller than the former; hence, $\hat{s}$ is practically smooth, see, e.g., Fig. 7. Setting $q \equiv \hat{\Delta}, p \equiv-\hat{s}$, for each fixed $Z$ (14) are the Hamilton equations (with "time" $\xi$ ) $q^{\prime}=\partial \check{H} / \partial p, p^{\prime}=-\partial \check{H} / \partial q$ of a system with Hamiltonian $\breve{H}(q, p, \xi ; Z) \equiv H(q,-p, \xi ; Z)$,

$$
\begin{aligned}
H(\Delta, s, \xi ; Z) & \equiv \gamma(s, \xi)+\mathcal{U}(\Delta ; Z), \quad \gamma(s, \xi) \equiv \frac{s^{2}+1+v(\xi)}{2 s} \\
\mathcal{U}(\Delta ; Z) & \equiv \frac{4 \pi e^{2}}{m c^{2}}[\tilde{\mathcal{N}}(Z+\Delta)-\tilde{\mathcal{N}}(Z)-\tilde{N}(Z) \Delta] \\
\tilde{\mathcal{N}}(Z) & \equiv \int_{0}^{Z} d Z^{\prime} \tilde{N}\left(Z^{\prime}\right)=\int_{0}^{Z} d Z^{\prime} \widetilde{n}_{0}\left(Z^{\prime}\right)\left(Z-Z^{\prime}\right) .
\end{aligned}
$$

Defining $\mathcal{U}$ we have fixed the free additive constant so that $\mathcal{U}(0, Z) \equiv 0$ for each $Z ; H-\sqrt{1+v}$ is positive definite. Below we shall abbreviate $P(\xi ; Z) \equiv(\hat{\Delta}(\xi ; Z), \hat{s}(\xi ; Z))$.

The right-hand side of $(14)_{2}$ is an increasing function of $\hat{\Delta}$, because so is $\tilde{N}(Z)$. As $v(\xi)$ is zero for $\xi \leq 0$ and positive for small $\xi>0$, then so are also $\hat{\Delta}(\xi, Z)$ and $\hat{s}(\xi, Z)-1$. Both keep increasing until $\hat{\Delta}$ reaches a positive maximum $\hat{\Delta}(\bar{\xi}, Z)$ at the $\xi=\bar{\xi}(Z)>0$ such that

$$
\hat{\Delta}^{\prime}(\bar{\xi}, Z)=0 \Leftrightarrow \hat{s}^{2}(\bar{\xi}, Z)=1+v(\bar{\xi})
$$

[note that $\bar{\xi}<l$ if $v(l)=0$ ]. We shall denote as $\zeta \equiv$ $\hat{\Delta}[\bar{\xi}(0), 0]$ the maximum penetration of the $Z=0$ electrons. For $\xi>\bar{\xi}(Z) \hat{\Delta}$ starts decreasing; $\hat{s}$ reaches a maximum at the $\xi=\xi_{r}(Z)$ such that $\hat{\Delta}\left(\xi_{r}, Z\right)=0$ (i.e. at $\xi=\xi_{r}(Z)$ the $Z$ electrons have regained their initial $z$ ). Both decrease for $\xi>\xi_{r}(Z)$, until $\hat{s}$ becomes so small, and the right-hand side of $(14)_{1}$ so large, that first $\hat{\Delta}$, and then $\hat{s}-1$, are forced to abruptly grow again to positive values. This prevents $\hat{s}$ from vanishing anywhere, consistently with (6). In $\xi$-intervals where $v(\xi) \equiv v_{c} \equiv$ const, $H$ is conserved, and all trajectories $P(\xi ; Z)$ in phase space (paths) are level curves 
$H(\Delta, s ; Z)=h(Z)$, above the line $s=0$, integrable by quadrature [25]. For $Z=0$ the paths are unbounded with $\hat{\Delta}(\xi, 0) \rightarrow-\infty$ as $\xi \rightarrow \infty$. For $Z>0$ the paths are cycles around the only critical point $C \equiv(\Delta, s)=\left(0, \sqrt{1+v_{c}}\right)$ (a center); therefore for $\xi \geq l v(\xi)=v(l)$, and these solutions are periodic. There exists a $Z_{b}>0$ such that: the paths $P(\xi ; Z)$ with $Z<Z_{b}$ cross the $\hat{\Delta}=-Z$ line twice, i.e. go out of the bulk and then come back into it; the path $P\left(\xi ; Z_{b}\right)$ is tangent to this line in the point $(\hat{\Delta}, \hat{s})=$ $\left(-Z_{b}, \sqrt{1+v(l)}\right)$ (where $\hat{\Delta}^{\prime}=0$ ); the paths $P(\xi ; Z)$ with $Z>Z_{b}$ do not cross this line. For $Z \leq Z_{b}$ let $\xi_{\text {ex }}(Z)$ be the first positive solution of the equation $\hat{\Delta}(\xi, Z)=-Z$, i.e. at $\xi=\xi_{\text {ex }}(Z)$ the $Z$ electrons exit the bulk:

$$
\hat{z}_{e}\left[\xi_{\mathrm{ex}}(Z), Z\right]=0 .
$$

The function $\xi_{\mathrm{ex}}(Z)$ is strictly increasing if $\partial_{Z} \hat{z}_{e}>0$.

For any family $P(\xi ; Z)$ of solutions of (14)-(15) let

$$
\begin{gathered}
\hat{u}^{z} \equiv \frac{1+v-\hat{s}^{2}}{2 \hat{s}}, \quad \hat{\gamma} \equiv \frac{1+v+\hat{s}^{2}}{2 \hat{s}}, \\
\hat{\boldsymbol{x}}_{e}(\xi, \boldsymbol{X})=\boldsymbol{X}+\hat{\boldsymbol{Y}}(\xi, Z), \quad \hat{\boldsymbol{Y}}(\xi, Z) \equiv \int_{0}^{\xi} d y \frac{\hat{\boldsymbol{u}}(y, Z)}{\hat{s}(y, Z)} \\
c \hat{t}(\xi, Z)=Z+\hat{\Xi}(\xi, Z), \\
\hat{\Xi}(\xi, Z) \equiv \int_{0}^{\xi} d y \frac{\hat{\gamma}(y, Z)}{\hat{s}(y, Z)}=\xi+\hat{\Delta}(\xi, Z)
\end{gathered}
$$

(note that $\hat{Y}^{z}=\hat{\Delta}$ ). The so defined $\hat{\boldsymbol{u}}, \hat{\gamma}, \hat{\boldsymbol{x}}_{e}$ are the solutions - expressed as functions of $\xi, X$ - of all equations and initial conditions [26]. Note that $\hat{\boldsymbol{x}}_{e}, \hat{t}$ can be obtained also solving the system of functional equations

$$
\xi=c t-z, \quad \hat{\Xi}(c t-z, Z)=c t-Z, \quad \boldsymbol{x}-\boldsymbol{X}=\hat{\boldsymbol{Y}}(c t-z, Z)
$$

[by (19) the second is actually equivalent to the $z$-component of the third] with respect to $t, x$. Clearly $\hat{\Xi}(\xi, Z)$ is strictly increasing and invertible with respect to $\xi$ for all fixed $Z$. Solving (20) with respect to $\xi, \boldsymbol{x}$ (resp. $\boldsymbol{\xi}, \boldsymbol{X}$ ) as functions of $t, \boldsymbol{X}$ (resp. of $t, \boldsymbol{x}$ ) and replacing the results in $\hat{\boldsymbol{u}}, \hat{\gamma}, \hat{s}, \ldots$ one obtains the solutions in the Lagrangian (resp. Eulerian) description: in particular one finds (generalizing [16])

$$
\begin{aligned}
\tilde{\xi}(t, Z) & =\hat{\Xi}^{-1}(c t-Z, Z), \quad \boldsymbol{x}_{e}(t, \boldsymbol{X})=\boldsymbol{X}+\hat{\boldsymbol{Y}}[\tilde{\xi}(t, Z), Z], \\
z_{e}(t, Z) & =Z+\hat{\Delta}[\tilde{\xi}(t, Z), Z]=c t-\tilde{\xi}(t, Z), \\
\tilde{s}_{e}(t, Z) & \equiv \hat{s}[\tilde{\xi}(t, Z), Z], \quad \tilde{\boldsymbol{u}}_{e}(t, Z)=\hat{\boldsymbol{u}}[\tilde{\xi}(t, Z), Z], \\
\boldsymbol{X}_{e}^{\perp}(t, \boldsymbol{x}) & =\boldsymbol{x}^{\perp}-\hat{\boldsymbol{Y}}^{\perp}\left[c t-z, Z_{e}(t, z)\right], \\
\boldsymbol{u}_{e}(t, z) & =\hat{\boldsymbol{u}}\left[c t-z, Z_{e}(t, z)\right] .
\end{aligned}
$$

Indeed, it is straightforward to check that $\left[z_{e}(t, Z), \tilde{s}_{e}(t, Z)\right]$ is the solution of (11)-(12) and $\boldsymbol{p}_{e}(t, \boldsymbol{x}) \equiv m c \boldsymbol{u}_{e}(t, z)$, $\boldsymbol{x}_{e}(t, \boldsymbol{X})$ of the PDE's (1) with the initial conditions $\boldsymbol{p}_{e}(0, \boldsymbol{X})=0, \boldsymbol{x}_{e}(0, \boldsymbol{X})=\boldsymbol{X}$ for $Z \geq 0$.

From (17), (18), (19) $)_{5}$, the times of maximal penetration and of expulsion of the $Z$ electrons are

$\bar{t}(Z)=\frac{Z+\hat{\Xi}(\bar{\xi}, Z)}{c}, \quad t_{\mathrm{ex}}(Z)=\frac{Z+\hat{\Xi}\left(\xi_{\mathrm{ex}}, Z\right)}{c}$.

Deriving (21) and the identity $y \equiv \hat{\Xi}\left[\hat{\Xi}^{-1}(y, Z), Z\right]$ we obtain a few useful relations, e.g.

$$
\begin{aligned}
\frac{\partial \hat{\Xi}^{-1}}{\partial Z} & =\left.\frac{-\hat{s}}{\hat{\gamma}} \frac{\partial \hat{\Delta}}{\partial Z}\right|_{\xi=\hat{\Xi}^{-1}(y, Z)}, \quad \frac{\partial z_{e}}{\partial Z}=\left.\frac{\hat{s}}{\hat{\gamma}} \frac{\partial \hat{z}_{e}}{\partial Z}\right|_{\xi=\hat{\Xi}^{-1}(c t-Z, Z)}, \\
\frac{\partial Z_{e}}{\partial z} & =\left.\frac{\hat{\gamma}}{\hat{s} \partial_{Z} \hat{z}_{e}}\right|_{(\xi, Z)=\left(c t-z, Z_{e}(t, z)\right)} .
\end{aligned}
$$

By (23), $\partial_{Z} \hat{z}_{e} \equiv 1+\partial_{Z} \hat{\Delta}>0$ is thus a necessary and sufficient condition for the invertibility of the maps $z_{e}: Z \mapsto z, \boldsymbol{x}_{e}: X \mapsto \boldsymbol{x}$ (at fixed $t$ ), justifying the hydrodynamic description of the plasma adopted so far and the presence of the inverse function $Z_{e}(t, z)$ in (21). Finally, from (4), (23) we find also

$$
n_{e}(t, z)=\left.\widetilde{n_{0}}\left[Z_{e}(t, z)\right] \frac{\hat{\gamma}}{\hat{s} \partial_{Z} \hat{z}_{e}}\right|_{(\xi, Z)=\left(c t-z, Z_{e}(t, z)\right)} .
$$

We can test the range of validity of the approximation $\boldsymbol{A}^{\perp}(t, z)=\boldsymbol{\alpha}^{\perp}(c t-z)$ by showing that the latter makes the modulus of the right-hand side of (9) much smaller than $\alpha^{\perp}(c t-z)$ on $D \equiv\left\{(t, z) \mid 0 \leq c t-z \leq \xi_{\text {ex }}\left(Z_{M}\right), 0 \leq c t+\right.$ $\left.z \leq \xi_{\text {ex }}\left(Z_{M}\right)\right\} \quad\left(Z_{M}\right.$ is defined below), or equivalently [multiplying by $e / m c^{2}$ and using (24)]

$$
\begin{gathered}
\text { for } x \equiv(t, z) \in D\left|\delta \boldsymbol{u}^{\perp}(t, z)\right| \ll\left|\boldsymbol{u}^{\perp}(c t-z)\right|, \\
\delta \boldsymbol{u}^{\perp}(t, z) \equiv \int_{D_{x} \cap T} d t^{\prime} d z^{\prime} \frac{2 \pi e^{2} \tilde{n}_{0}\left[Z_{e}\left(t^{\prime}, z^{\prime}\right)\right] \boldsymbol{u}^{\perp}\left(c t^{\prime}-z^{\prime}\right)}{m c\left[\hat{s} \partial_{Z} \hat{z}_{e}\right]_{(\xi, Z)=\left(c t^{\prime}-z^{\prime}, Z_{e}\left(t^{\prime}, z^{\prime}\right)\right)}}
\end{gathered}
$$

actually, it suffices to check this inequality on the worldlines of the expelled electrons.

\section{B. Auxiliary problem: constant initial density}

As a simplest illustration of the approach, and for later application to a step-shaped initial density, we first consider the case that $\widetilde{n_{0}}(Z)=n_{0}$. Then $F_{e}^{z}$ is the force of a harmonic oscillator (with equilibrium at $z_{e}=Z$ ) $F_{e}^{z}\left(z_{e}, Z\right)=$ $-4 \pi n_{0} e^{2}\left[z_{e}-Z\right]=-4 \pi n_{0} e^{2} \Delta$; the $Z$-dependence disappears completely in (14)-(15), which reduces to the auxiliary Cauchy problem 

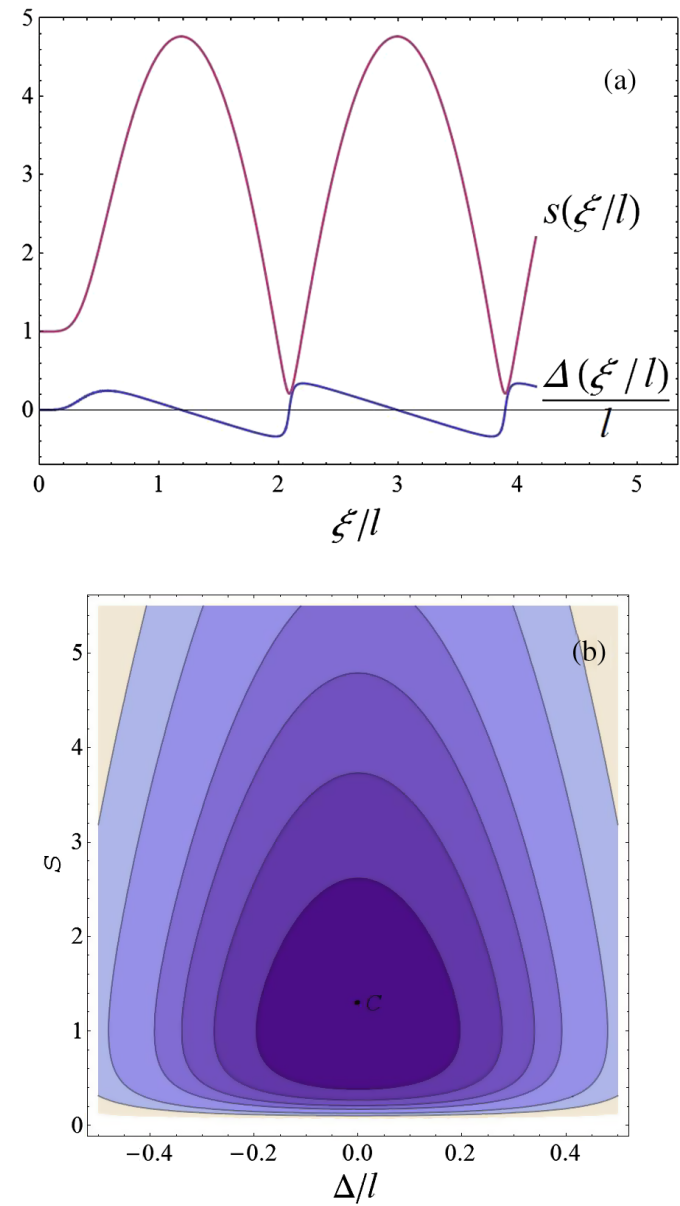

FIG. 3. (a) Solution of (26) for $M l^{2}=26$ and the $v(\xi)$ as in Sect. IV of average intensity $I=10^{19} \mathrm{~W} / \mathrm{cm}^{2}$. (b) Paths $P(\xi ; Z)$ around the center $C$ for $M l^{2}=26, v_{c}=0$.

$\Delta^{\prime}=\frac{1+v}{2 s^{2}}-\frac{1}{2}, \quad s^{\prime}=M \Delta, \quad \Delta(0)=0, \quad s(0)=1$,

where $M \equiv 4 \pi e^{2} n_{0} / m c^{2}$. The potential energy in (16) takes the form $\mathcal{U}(\Delta, Z) \equiv M \Delta^{2} / 2$. Problem (26), and hence also its solution $[\Delta(\xi), s(\xi)]$, the value of the energy as a function of $\xi$ and the functions defined in (19), are $Z$-independent. It follows $\partial_{Z} \hat{\Delta} \equiv 0$ and by (23) the automatic invertibility of $z_{e}(t, Z)$; moreover, the inverse function $Z_{e}(t, z)$ has the closed form

$$
Z_{e}(t, z)=c t-\Xi(c t-z)=z-\Delta(c t-z)
$$

[here $\Xi(\xi) \equiv \xi+\Delta(\xi)$ ], what makes the solutions (21) of the system of functional equations (20), as well as those of (1), completely explicit in terms of $\Xi$ and the inverse $\Xi^{-1}$ only. As a consequence, all Eulerian fields depend on $t, z$ only through $c t-z$ (i.e. evolve as traveling-waves). In Fig. 3-left we plot some solution of (26). If $v(\xi) \equiv v_{c} \equiv$ const all paths $P(\xi ; Z)$ are cycles around $C$ (Fig. 3-right), corresponding to periodic solutions. Within the bulk electron
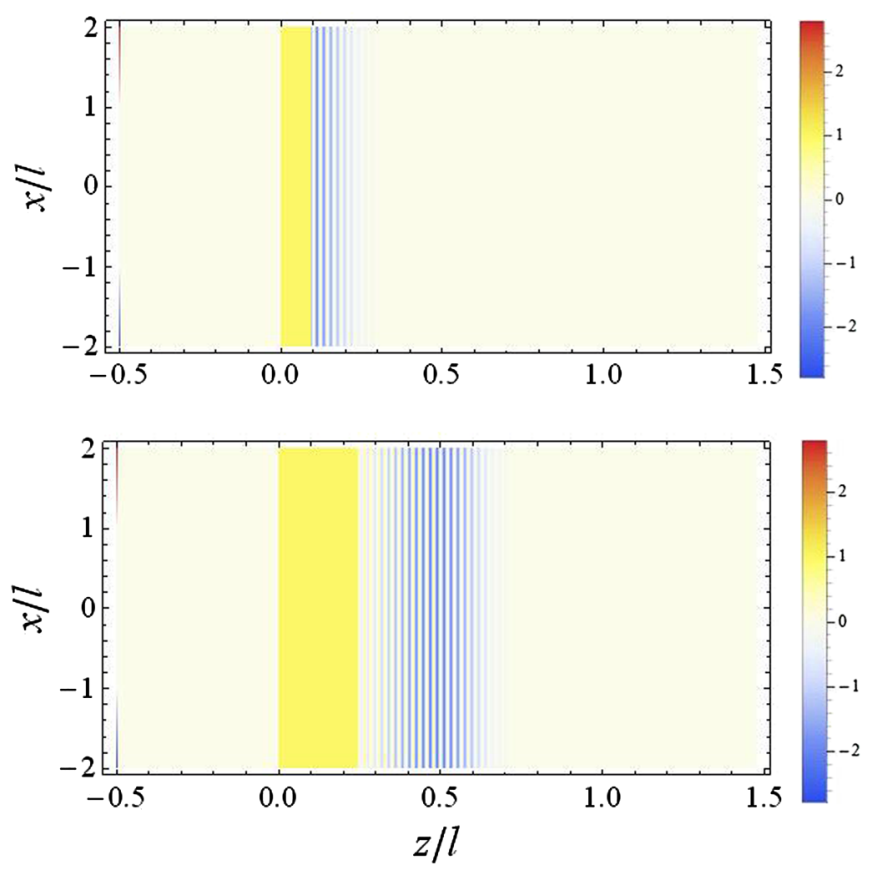

FIG. 4. Normalized charge density plot $1-n_{e} / n_{0}$ under the same conditions as in Fig. 7 after about 25 fs (up) and $70 \mathrm{fs}$ (down), i.e. resp. before and after the maximal penetration time $\bar{t}(0)=51 \mathrm{fs}$; in the latter picture the electrons travelling backwards make light yellow-blue striped the region between the yellow and the white-blue striped ones.

trajectories for slowly modulated laser pulse like the ones considered in Sec. IV are typically as plotted in Fig. 8; in average they have no transverse drift, but a longitudinal forward/backward one. Figure 4 shows a couple of corresponding charge density plots.

\section{3-DIMENSIONAL EFFECTS}

We now discuss the effects of the finiteness of $R$. For brevity, for any nonnegative $r, L$ we shall denote as $C_{r}$ the infinite cylinder of equation $\rho \leq r$, as $C_{r}^{L}$ the cylinder of equations $\rho \leq r, 0 \leq z \leq L$. The ponderomotive force of the pulse will boost forward (as in Fig. 8) only the small- $Z$ electrons located within (or nearby) $C_{R}$. These forward boosted electrons (FBE) will be thus completely expelled out of a cylinder which will reach its maximal extension $C_{R}^{\zeta}$ around the time $\bar{t}(0)$ of maximal longitudinal penetration $\zeta \equiv \hat{\Delta}[\bar{\xi}(0), 0]$ of the $Z=0$ electrons. The displaced charges modify $\boldsymbol{E}$. By causality (see Appendix A), for $\boldsymbol{x}$ near the $\vec{z}$ axis $\boldsymbol{E}(t, \boldsymbol{x})$ is the same as in the plane wave case for $t \lesssim \bar{t}(0)+R / c$, and smaller afterwards. We choose $\widetilde{n_{0}}$, $R$ so that they fulfill

$\frac{\left[t_{\mathrm{ex}}-\bar{t}\right] c}{R} \sim 1, \quad r \equiv R-\frac{\zeta\left(t_{\mathrm{ex}}-l / c\right)}{2\left(t_{\mathrm{ex}}-\bar{t}\right)} \theta\left(c t_{\mathrm{ex}}-l\right)>0$ 
and condition (25) for all $x=(t, \boldsymbol{x})$ such that $t \lesssim \bar{t}(0)+R / c$; here $\bar{t} \equiv \bar{t}(0), t_{\mathrm{ex}} \equiv t_{\mathrm{ex}}(0)$ are the times of maximal penetration and of expulsion from the bulk of the $Z=0$ electrons [see (22)]. [As $\widetilde{n_{0}}$ grows from zero the right-hand side of (25) does as well, whereas $\bar{t}(0), t_{\mathrm{ex}}(0)-$ $\bar{t}(0)$ decrease]. In Appendix A we show that conditions (28) respectively ensure that these FBE, at least within an inner cylinder $\rho \leq r \leq R$ : (i) move approximately as in Sec. II until their expulsion; (ii) are expelled before lateral electrons (LE), which are initially located outside the surface of $C_{R}$ and are attracted towards the $\vec{z}$-axis (see Fig. 1(c)), obstruct their way out. For the validity of our model we must a posteriori check also that the expelled $C_{r}$ electrons remain in $C_{R}$,

i.e. their transverse oscillations $\Delta x_{e}$ are $\ll R$.

In the plane model the expelled $Z>0$ electrons cannot escape to $z \rightarrow-\infty$ because they are decelerated by the constant electric force $\tilde{F}_{e}^{z}(t, Z)=4 \pi e^{2} \tilde{N}(Z)>0$, see (10). The real electric force $\widetilde{F_{e}^{z r}}>0$ acting on the $C_{r}$ electrons after expulsion is generated by charges localized in $C_{R}$; hence $\widetilde{F_{e}^{z r}} \propto 1 / z_{e}^{2}$ as $z_{e} \rightarrow-\infty$, and the escape of expelled electrons is no more excluded a priori. Moreover, since $\widetilde{F_{e}^{z}}(t, 0)=0$, it should be also $\widetilde{F_{e}^{z r}}(t, 0)=0$, allowing the escape of the $Z=0$ electrons; by continuity there will exist some positive $Z_{M} \leq Z_{b}$ such that the $C_{r}^{Z_{M}}$ electrons escape to infinity. We stick to the estimate $\widetilde{F_{e}^{z r}}$ on the $\vec{z}$-axis electrons; we assume that after the pulse has overcome them, they move along the $\vec{z}$-axis. Actually this will be justified below if $\hat{u}^{\perp}(l) \simeq 0$, which in turn holds if, as usual, $l \gg \lambda$ [see the comments after (33)]. In Fig. 5(a) we schematically depict the charge distribution expected shortly after the expulsion. The light blue area is occupied only by the $X \in C_{r}^{Z_{M}}$ electrons. The orange area is positively charged due to an excess of ions. For any $Z$-electrons moving along the $\vec{z}$-axis consider the surfaces $S_{0}, S_{1}, S_{2}$ occupied at time $t$ by the $X^{\prime} \in C_{r}$ electrons respectively having $Z^{\prime}=0, Z, Z_{2}(Z)$, where $Z_{2}(Z)$ is defined by the condition $\tilde{N}\left(Z_{2}\right)=2 \tilde{N}(Z)$, which ensures that the electron charges contained between $S_{0}, S_{1}$ and $S_{1}$, $S_{2}$ are equal (in the figure $S_{0}, S_{1}, S_{2}$ are respectively represented by the left border of the blue area, the dashed line and the solid line). The longitudinal electric force $\widetilde{F_{e}^{z r}}$ acting at time $t$ on this $Z$-electron is nonnegative and can be decomposed and bound as follows [13]:

$0 \leq \widetilde{F_{e}^{z r}}(t, Z)=-e \tilde{E_{-}^{z}}(t, Z)-e \tilde{E_{+}^{z}}(t, Z) \leq F_{e r}^{z}[\tilde{\Delta}(t, Z), Z]$.

Here $\tilde{E_{-}^{z}}(t, Z)$ stands for the part of the longitudinal electric field generated by the electrons between $S_{0}, S_{2}$; since those between $S_{0}, S_{1}$ have by construction the same charge as those between $S_{1}, S_{2}$, but are more dispersed, it will be
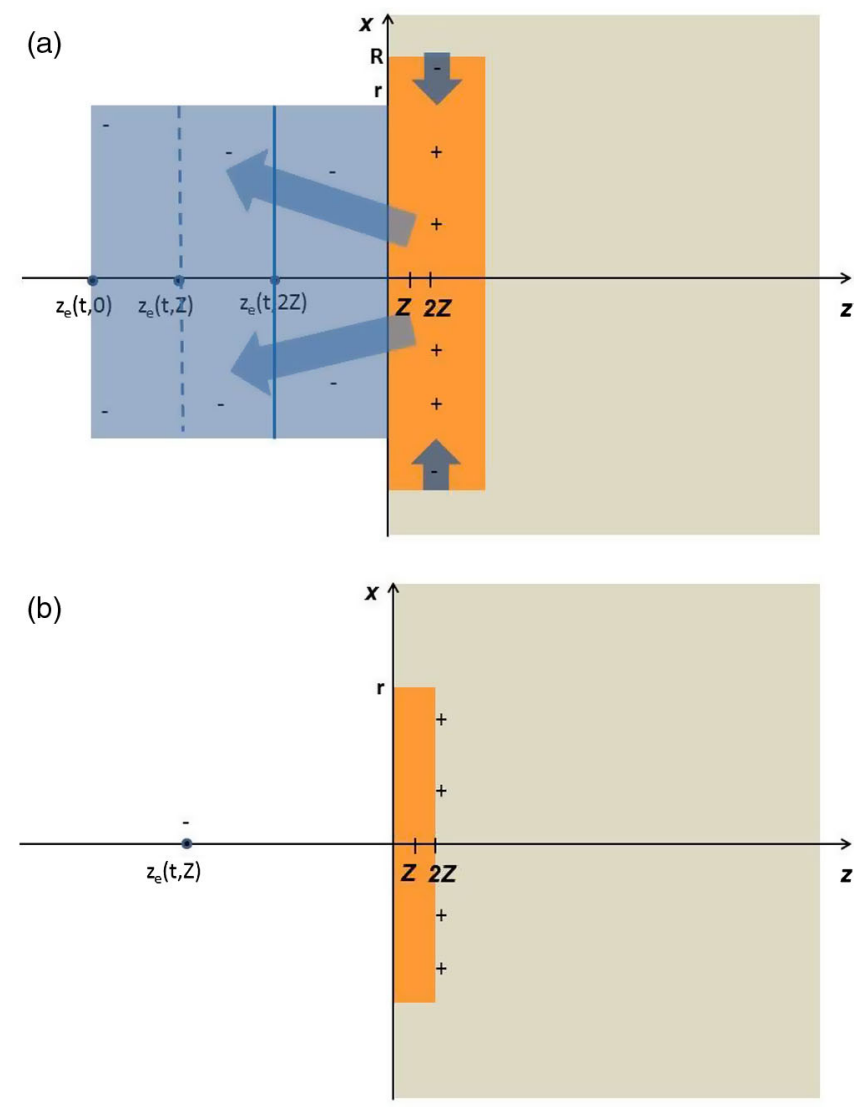

FIG. 5. (a) schematic picture of the expected charge distribution shortly after the expulsion (long arrows) of surface electrons; short arrows represent the inward motion of the lateral electrons; (b) simplified charge distribution generating the effective potential energy.

$-e \widetilde{E_{-}^{z}}(t, Z) \leq 0$. The part $-e \widetilde{E_{+}^{z}}(t, Z)$ of $\widetilde{F_{e}^{z r}}$ generated by the ions and the remaining electrons (at the right of $S_{2}$ ) will be smaller than the force $F_{e r}^{z}$ generated by the charge distribution of Fig. 5(b), where the remaining electrons are located farther from $\left(0,0, z_{e}\right)$ (in their initial positions $\boldsymbol{X}^{\prime}$, not in the ones at $t$ ) and hence generate a smaller repulsive force. This explains the second inequality in the equation. In Appendix B we show that for $z_{e} \equiv Z+\Delta \leq 0$

$$
\frac{F_{e r}^{z}(\Delta, Z)}{2 \pi e^{2}}=2 \tilde{N}(Z)-\int_{0}^{Z_{2}(Z)} d Z^{\prime} \frac{\widetilde{n_{0}}\left(Z^{\prime}\right)\left(Z^{\prime}-z_{e}\right)}{\sqrt{\left(Z^{\prime}-z_{e}\right)^{2}+r^{2}}} .
$$

Commendably, $F_{e r}^{z}$ is conservative, nonnegative and goes to zero as $\Delta \rightarrow-\infty$, while it reduces to zero for $Z=0$ and to $4 \pi e^{2} \tilde{N}(Z)$ as $r \rightarrow \infty$, as $\widetilde{F_{e}^{z}}$ in $(10)_{3}$; it becomes a function of $t$ (resp. $\xi$ ) through $\tilde{\Delta}(t, Z)$ [resp. $\hat{\Delta}(\xi, Z)$ ] only. We therefore modify the dynamics outside the bulk replacing $F_{e}^{z}$ by $F_{e r}^{z}$, or equivalently $\mathcal{U}$ by $\mathcal{U}_{r}$ in (16), where $\mathcal{U}_{r}$ is continuous and equals $\mathcal{U}$ for $z_{e} \equiv Z+\Delta \geq 0$, and the potential energy (B2) associated to $F_{e r}^{z}$ for $z_{e} \equiv Z+\Delta \leq 0$; there $\mathcal{U}_{r}$ is a decreasing function of $\Delta$ 

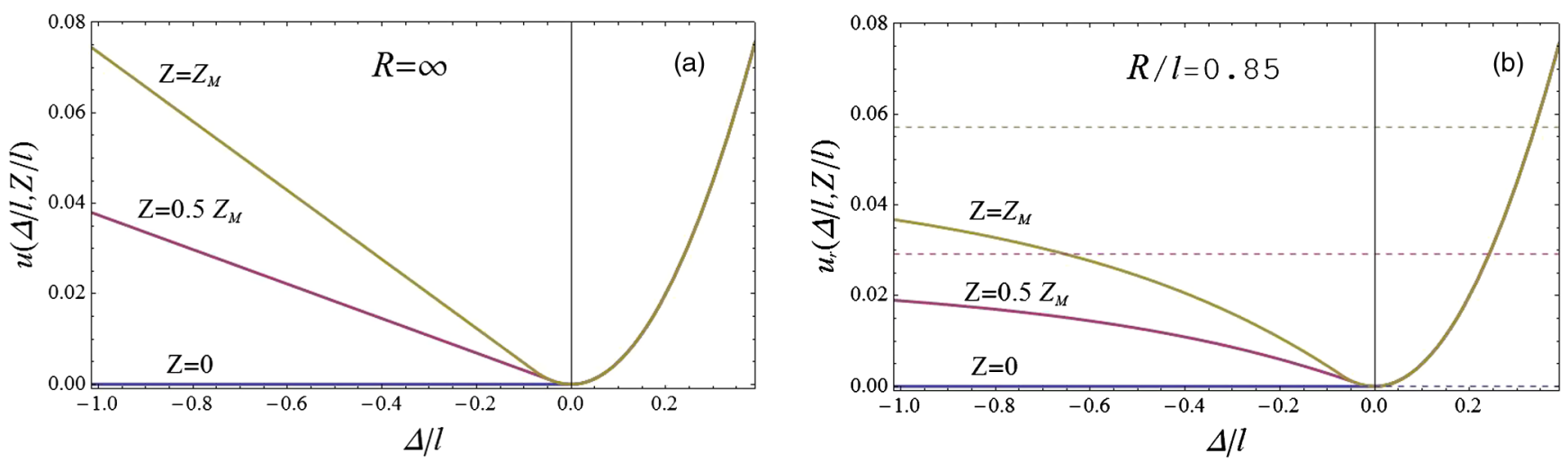

FIG. 6. Rescaled longitudinal electric potential energies $u \equiv \mathcal{U} / 4 \pi n_{0} e^{2} l^{2}, u_{r} \equiv \mathcal{U}_{r} / 4 \pi n_{0} e^{2} l^{2}$ for (a) idealized plane wave $R / l=\infty$ or (b) for $R / l=0.85$, plotted as functions of $\Delta$ for $Z / Z_{M}=0, .2, .4, .6, .8,1$; the horizontal dashed lines are the left asymptotes of $u_{r}$ for the same values of $Z / Z_{M}$. Here the initial electron density is step-shaped: $\widetilde{n_{0}}(Z)=n_{0} \theta(Z)$.

with finite left asymptotes (B3). We will thus underestimate the final energy of the electrons, because $F_{e r}^{z}$ is larger than the real electric force $\widetilde{F_{e}^{z r}}$ decelerating the electrons outside the bulk; this makes our estimates safer. In Fig. 6 we plot suitably rescaled $\mathcal{U}$ and $\mathcal{U}_{r}$ for $\widetilde{n_{0}}(Z)=n_{0} \theta(Z)$. After the pulse is passed we can compute $\gamma$ as a function of $\Delta, Z$ using energy conservation $m c^{2} \gamma+\mathcal{U}_{r}(\Delta, Z)=$ const. For the expelled electrons the final relativistic factor $\gamma_{f}(Z) \equiv \gamma_{e}(\Delta=-\infty, Z)$ is the decreasing function (B4). The maximum of $\gamma_{f}(Z)$ is $\gamma_{M} \equiv \gamma_{f}(0)$. Let $Z_{M} \leq Z_{b}$ be the $Z$ fulfilling $\gamma_{f}(Z)=1$. The estimated total number $N_{e}$, electric charge (in absolute value) $Q$, and kinetic energy $\mathrm{E}$ of the $X \in C_{r}^{Z_{M}}$ escaped electrons are thus

$$
\begin{aligned}
N_{e} & \sim \pi r^{2} \tilde{N}\left(Z_{M}\right), \quad Q \sim e N_{e}, \\
\mathrm{E} & \sim m \pi c^{2} r^{2} \int_{0}^{Z_{M}} d Z \widetilde{n_{0}}(Z)\left[\gamma_{f}(Z)-1\right] .
\end{aligned}
$$

The number of escaped $\boldsymbol{X}^{\prime} \in C_{r}^{Z_{M}}$ electrons with $Z \leq Z^{\prime} \leq$ $Z+d Z$ is estimated as $\pi r^{2} \widetilde{n_{0}}(Z) d Z$, that with relativistic factor between $\gamma$ and $\gamma+d \gamma$ is estimated as $d N=$ $\pi r^{2}\left[\widetilde{n_{0}}(Z) /\left|d \gamma_{f} / d Z\right|\right]_{Z=\hat{Z}(\gamma)} d \gamma$, where $\hat{Z}(\gamma)$ is the inverse of $\gamma_{f}(Z)$ (a strictly decreasing function, see Appendix B). Hence the fraction of escaped electrons with final relativistic factor between $\gamma$ and $\gamma+d \gamma$ is estimated as $\nu(\gamma) d \gamma$, where

$$
\nu(\gamma) \equiv \frac{1}{N_{e}} \frac{d N}{d \gamma}=\left.\frac{1}{\tilde{N}\left(Z_{M}\right)} \frac{\widetilde{n_{0}}(Z)}{\left|d \gamma_{f} / d Z\right|}\right|_{Z=\hat{Z}(\gamma)}
$$

determines the associated energy spectrum. As $\alpha^{\perp}(\xi)=$ $\alpha^{\perp}(l)$ if $\xi \geq l$, by (7) the final transverse deviation of the escaped electrons will be

$$
\frac{\beta_{f}^{\perp}}{\beta_{f}^{z}}(Z)=\frac{u_{f}^{\perp}}{u_{f}^{z}}(Z)=\frac{u_{f}^{\perp}}{\sqrt{\gamma_{f}^{2}(Z)-1-u_{f}^{\perp 2}}}
$$

where $u_{f}^{\perp} \equiv \hat{u}^{\perp}(l)$. This is an increasing function of $Z$, because $\gamma_{f}(Z)$ is decreasing. If $\lambda \ll l$ then $u_{f}^{\perp} \simeq 0$ (see next section), and (33) is negligible unless $Z \simeq Z_{M}$.

\section{A. Step-shaped initial density}

If $\widetilde{n_{0}}(Z)=n_{0} \theta(Z)$ then $\tilde{N}(Z)=n_{0} \theta(Z) Z$, and for $Z \geq 0$

$$
\frac{F_{e r}^{z}(\Delta, Z)}{2 \pi n_{0} e^{2}}= \begin{cases}-2 \Delta(\text { elastic force }), & z_{e}>0 \\ 2 Z+\sqrt{(Z+\Delta)^{2}+r^{2}}-\sqrt{(Z-\Delta)^{2}+r^{2}}, & z_{e} \leq 0\end{cases}
$$

Since the first expression is the same as in the case $\widetilde{n_{0}}(Z)=n_{0}$, the motion of the $Z$-electron will be as in subsection II B until $\xi=\xi_{\text {ex }}(Z)$. The second expression goes to the constant force $4 \pi n_{0} e^{2} Z$ as $r \rightarrow \infty$, as expected. The motion for $\xi>\xi_{\text {ex }}(Z)$ will be studied in detail in [25]; we plot the graphs of a typical solution (until the expulsion) in Fig. 7 and a few corresponding electron trajectories in Fig. 8. We can readily understand that it will be
$\partial_{Z} \hat{z}_{e}(\xi, Z)>0$ for all $\xi$ and $0 \leq Z \leq Z_{M}$, since this holds for $\xi \leq \xi_{\mathrm{ex}}(Z)$ [by the comments following (26)], and both $\xi_{\mathrm{ex}}(Z)$ and the decelerating force $F_{e r}^{z}(\Delta, Z)$ (outside the bulk) increase with $Z$, while the speed of exit from the bulk decreases with $Z$, whence the distance between electrons with different $Z$ increases with $\xi, t$. The $Z_{b}$ introduced before (18) is now the solution of the equation $\sqrt{1+v(l)}+M Z_{b}^{2} / 2=h$, i.e., the $Z$ corresponding to 

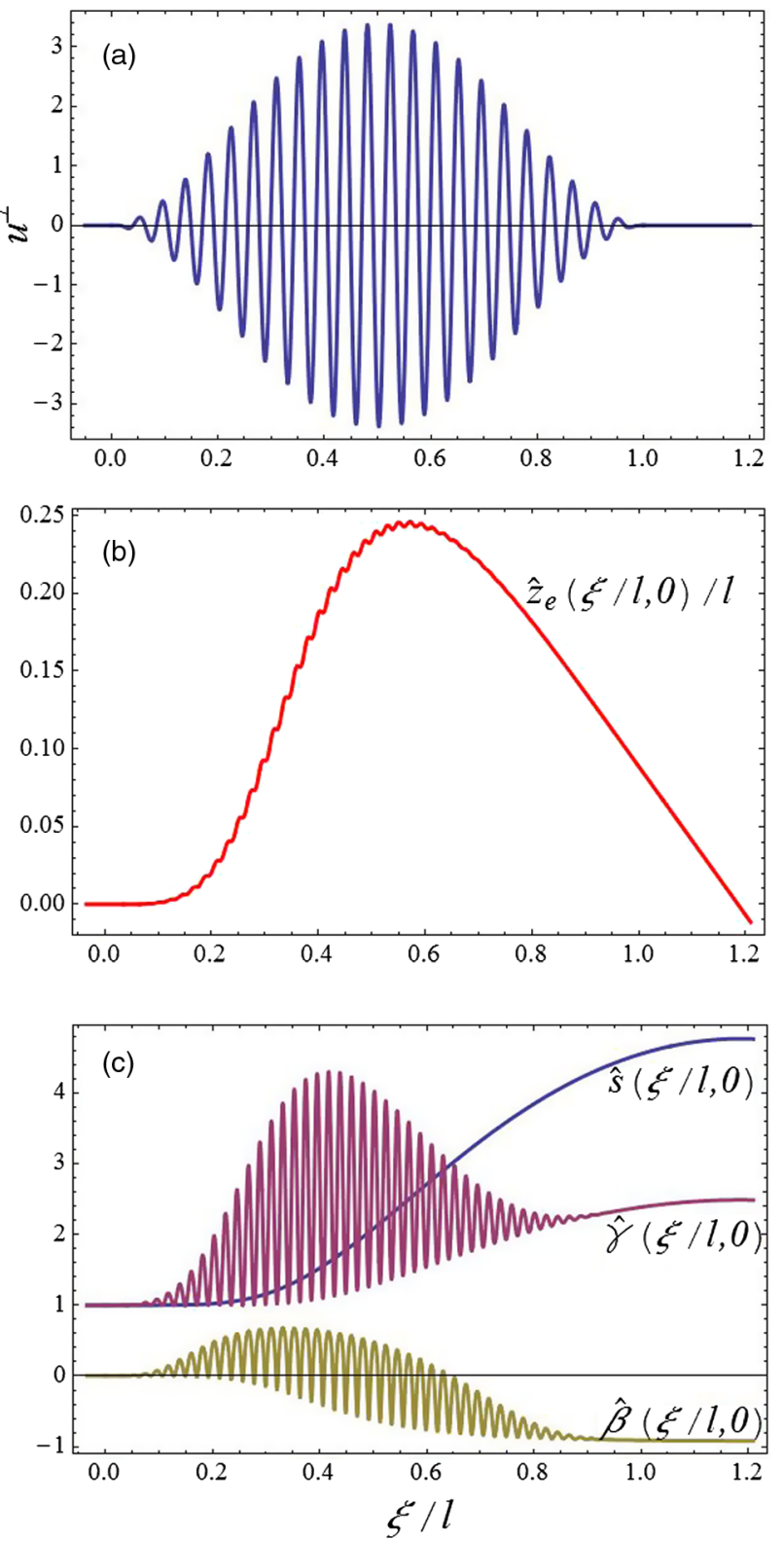

FIG. 7. (a) Laser pulse of average intensity $I=10^{19} \mathrm{~W} / \mathrm{cm}^{2}$ and shape as in Sec. IV, with $l=18.75 \mu \mathrm{m}$. (b-c) Corresponding solution of (14)-(15) for initial density $\widetilde{n_{0}}(Z)=n_{0} \theta(Z)$, with $n_{0}=21 \times 10^{17} \mathrm{~cm}^{-3}$ (i.e. $M l^{2}=26$ ).

the zero longitudinal velocity and the final value of the energy $h$ after the interaction of the pulse; one can determine $h$ evaluating $H$ at $\xi=l, h=\frac{1}{2}\{s(l)+[1+v(l)] /$ $\left.s(l)+M[\Delta(l)]^{2}\right\}$. Hence,

$$
Z_{b}=\sqrt{[\Delta(l)]^{2}+[s(l)-\sqrt{1+v(l)}]^{2} / 2 M s(l)} .
$$

$\gamma_{f}(Z), \nu(\gamma)$ admit rather explicit forms (B7), (B8). In Sec. IV we plot spectra $\nu(\gamma)$ corresponding to several $n_{0}$ and intensities. Moreover, $Q=\pi r^{2} e n_{0} Z_{M}$,
$\mathrm{E}=\pi r^{2} n_{0} m c^{2} \int_{0}^{Z_{M}} d Z\left[\gamma_{f}(Z)-1\right]$. Finally, if $\xi_{\mathrm{ex}}(0)<l$ then $\delta \boldsymbol{u}^{\perp}$ in (25) becomes

$$
\delta \boldsymbol{u}^{\perp}(t, z)=\frac{M}{2} \int_{0}^{c t-z} d \xi^{\prime} \frac{\hat{\boldsymbol{u}}^{\perp}\left(\xi^{\prime}\right)}{s\left(\xi^{\prime}\right)} \int_{0}^{c t+z} d \xi^{\prime \prime} \theta\left[\frac{\xi^{\prime}+\xi^{\prime \prime}}{2}-\Xi\left(\xi^{\prime}\right)\right] .
$$

\section{NUMERICAL RESULTS}

We assume for simplicity that the pulse is a slowly modulated sinusoidal function linearly polarized in the $x$ direction: $\boldsymbol{\epsilon}^{\perp}(\xi)=\epsilon_{S}(\xi) \hat{\boldsymbol{x}} \cos k \xi$, the modulating amplitude $\epsilon_{s}(\xi) \geq 0$ is nonzero only for $0<\xi<l$, and slowly varies on the scale of the period $\lambda \equiv 2 \pi / k \ll l$, i.e. $\lambda\left|\epsilon_{s}{ }^{\prime}\right| \ll\left|\epsilon_{s}\right|$ on the support of $\epsilon_{s}$. Integrating by parts we find $\alpha^{\perp}(\xi)=$ $\hat{\boldsymbol{x}} \epsilon_{s}(\xi)(\sin k \xi) / k+O\left(1 / k^{2}\right)$ [23] and, in terms of the rescaled amplitude $w(\xi) \equiv e \epsilon_{s}(\xi) / k m c^{2}$,

$\hat{\boldsymbol{u}}^{\perp}(\xi) \simeq \hat{\boldsymbol{x}} w(\xi) \sin (k \xi), \quad v(\xi) \simeq w^{2}(\xi) \sin ^{2}(k \xi)$,

where $a \simeq b$ means $a=b+O\left(1 / k^{2}\right)$. Note that, as $\epsilon_{s}(\xi)=0$ for $\xi \geq l$, this implies $u_{f}^{\perp}=\hat{u}^{\perp}(l) \simeq 0$, as anticipated.

If we approximate as $\chi_{R}(\rho) \equiv \theta(R-\rho)$ the cutoff function in (3), the average pulse intensity on its support is $I=c \mathcal{E} / \pi R^{2} l$. Here $\mathcal{E}$ is the EM energy carried by the pulse,

$\mathcal{E}=\int_{V} d V \frac{\boldsymbol{E}^{\perp 2}+\boldsymbol{B}^{\perp 2}}{8 \pi} \simeq \frac{R^{2}}{4} \int_{0}^{l} d \xi \boldsymbol{\epsilon}^{\perp 2}(\xi) \simeq \frac{R^{2}}{8} \int_{0}^{l} d \xi \epsilon_{s}^{2}(\xi)$.

High power lasers produce pulses where $\lambda \sim 1 \mu \mathrm{m}$ and $\epsilon_{s}$ is approximately Gaussian, $\epsilon_{s}(\xi) \propto \exp \left[-\left(\xi-\xi_{0}\right)^{2} / 2 \sigma\right] ; \sigma$ is related to the fwhm (full width at half maximum) $l^{\prime}$ of $\epsilon_{s}^{2}$ by $\sigma=l^{2} / 4 \ln 2$. If initially matter is composed of atoms then $\epsilon_{s}(c t-z)$ can be considered zero where it is under the ionization threshold, because the pulse has not converted matter into a plasma yet. Hence we adopt as a modulating amplitude $\epsilon_{s}(\xi)$ the cutoff Gaussian

$$
\begin{aligned}
\epsilon_{g}(\xi) & =b_{g} \exp \left[\frac{-(\xi-l / 2)^{2}}{2 \sigma}\right] \theta(\xi) \theta(l-\xi), \quad \sigma=\frac{l^{\prime 2}}{4 \ln 2}, \\
b_{g}^{2} & =\frac{16 \sqrt{\ln 2}}{\sqrt{\pi}} \frac{\mathcal{E}}{R^{2} l^{\prime}}, \quad l^{2}=\frac{l^{\prime 2}}{\sqrt{\ln 2}} \ln \left[\frac{\sqrt{\ln 2} m c^{2} \mathcal{E}(e \lambda)^{2}}{U_{i} \sqrt{\pi} l^{\prime}\left(\pi R m c^{2}\right)^{2}}\right],
\end{aligned}
$$

where $U_{i}$ is the first ionization potential (for Helium $U_{i} \simeq 24 \mathrm{eV}$ ); the formula for $b_{g}^{2}$ follows replacing the Ansatz (39) ${ }_{1}$ in (38) [neglecting the tails left out by the 


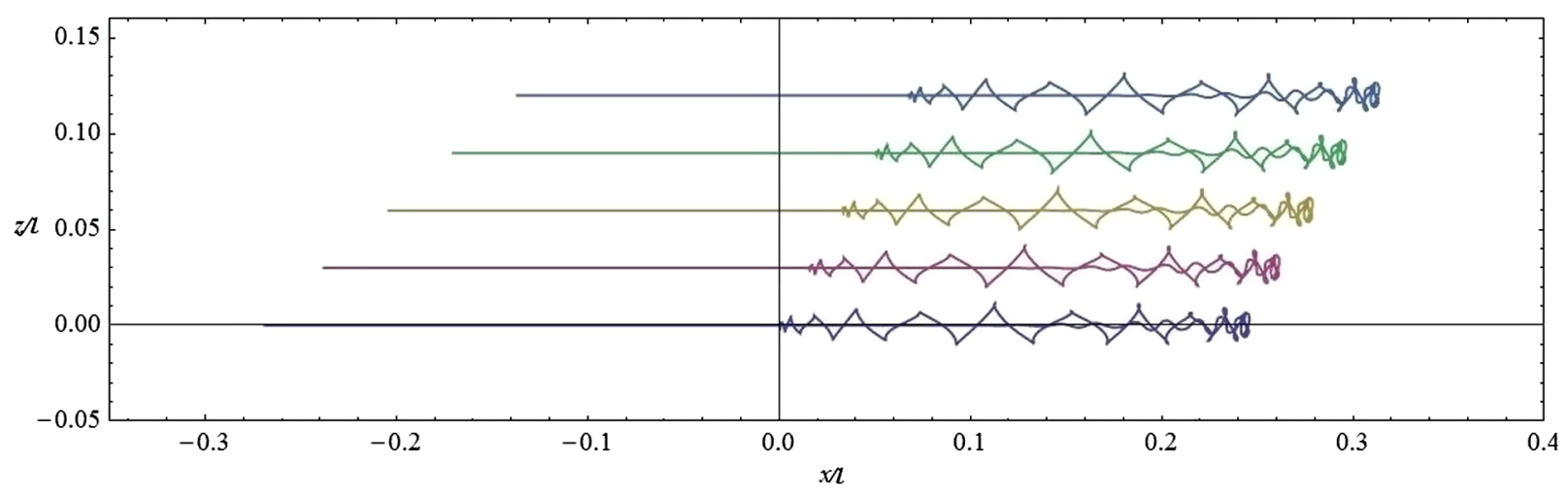

FIG. 8. Trajectories gone in ca. 150 fs by electrons initially located at $Z / Z_{M}=0,0.25,0.5,0.75,1$ under conditions as in Fig. 7 .

cutoff $\theta(\xi) \theta(l-\xi)]$. Numerical computations are easier if we adopt [16] as $\epsilon_{s}(\xi)$ the following cutoff polynomial:

$$
\epsilon_{p}(\xi) \equiv \frac{b_{p}}{4}\left[1-\left(2 \xi / l_{p}-1\right)^{2}\right]^{2} \theta(\xi) \theta\left(l_{p}-\xi\right),
$$

$b_{p}, l_{p}$ are determined by the requirement to lead to the same fwhm and $\mathcal{E}: b_{p}^{2}=5040 \mathcal{E} / R^{2} l_{p}$ and $l_{p}=5 l^{\prime} / 2$.

We now present the results of extensive numerical simulations based on the experimental parameters available already now at the FLAME facility [27] or in the near future at the ILIL facility [28]: $l^{\prime} \simeq 7.5 \mu \mathrm{m}$ (implying $l_{p}=18.75 \mu \mathrm{m}$ ), $\lambda \simeq 0.8 \mu \mathrm{m}$ (implying $k l_{p}=2 \pi l_{p} / \lambda \simeq 147$ ), $\mathcal{E}=5 \mathrm{~J}$, and $R$ tunable by focalization in the range $10^{-4} \div 1 \mathrm{~cm}$. We model the electron density: first as the step-shaped one $\widetilde{n_{0}}(Z)=n_{0} \theta(Z)$ (this allows analytical derivation of more results); then as a function smoothly increasing from zero to the asymptotic value $n_{0}$, with substantial variation in the interval $0 \leq Z \leq L \equiv 20 \mu \mathrm{m}$ (as motivated by experiments, see Sec. V), more precisely $\widetilde{n_{0}}(Z)=n_{0} \theta(Z) \tanh (Z / L)$. We have numerically solved the corresponding systems (14)-(15) and proceeded as in Sec. III, for $R=16,15,8,4,2,1 \mu \mathrm{m}$ [resp. leading to average intensities $\left.I / 10^{19}\left(\mathrm{~W} / \mathrm{cm}^{2}\right) \simeq 1,1.1,4,16,64,255\right]$, $n_{0}$ in the range $10^{17} \mathrm{~cm}^{-3} \leq n_{0} \leq 3 \times 10^{20} \mathrm{~cm}^{-3}$ and $Z \leq Z_{M}$; all results follow from these solutions.

In Fig. 9-left we plot the maximal final relativistic factor $\gamma_{M}$ of the expelled electrons as a function of $n_{0}$, with the above values of $I$ and $\widetilde{n_{0}}(Z)=n_{0} \theta(Z)$; each graph stops where $n_{0}$ becomes too large for conditions $(25),(28)_{1}$, or (29) to be fulfilled and is red where condition $(28)_{2}$ is no more fulfilled. The latter prevents collisions with the LE and becomes superfluous if the target is a solid cylinder of radius $R$ (since then there are no LE) [29]; the $I=$ $64,255 \times 10^{19} \mathrm{~W} / \mathrm{cm}^{2}$ graphs are plot green for densities corresponding to the lightest solids (aerogels) available today. As expected [13]: 1) as $n_{0} \rightarrow 0 \gamma_{M}-1 \propto n_{0} I^{2}$; 2) each graph $\gamma_{M}\left(n_{0} ; I\right)$ has a unique maximum $\gamma_{M M}(I) \equiv$ $\gamma_{M}\left(n_{0 M} ; I\right)$ at $n_{0 M} \sim \bar{n}_{0}$, where $\bar{n}_{0}$ is the density making $\bar{\xi}(0)=l / 2$, namely such that the $Z=0$ electrons reach the maximal penetration $\zeta=\hat{\Delta}(l / 2,0)$ when they are reached by the pulse maximum. The dependence of $\gamma_{M}$ on $n_{0}$ is anyway rather slow. The striking $\gamma_{M M}(I) \propto I$ behavior shown in Fig. 9 up-center hints at scaling laws and will be discussed elsewhere. In Fig. 10 we plot sample spectra $\nu(\gamma)$ for $I / 10^{19}\left(\mathrm{~W} / \mathrm{cm}^{2}\right) \simeq 1,4,16,64$ and $\widetilde{n_{0}}$ compatible
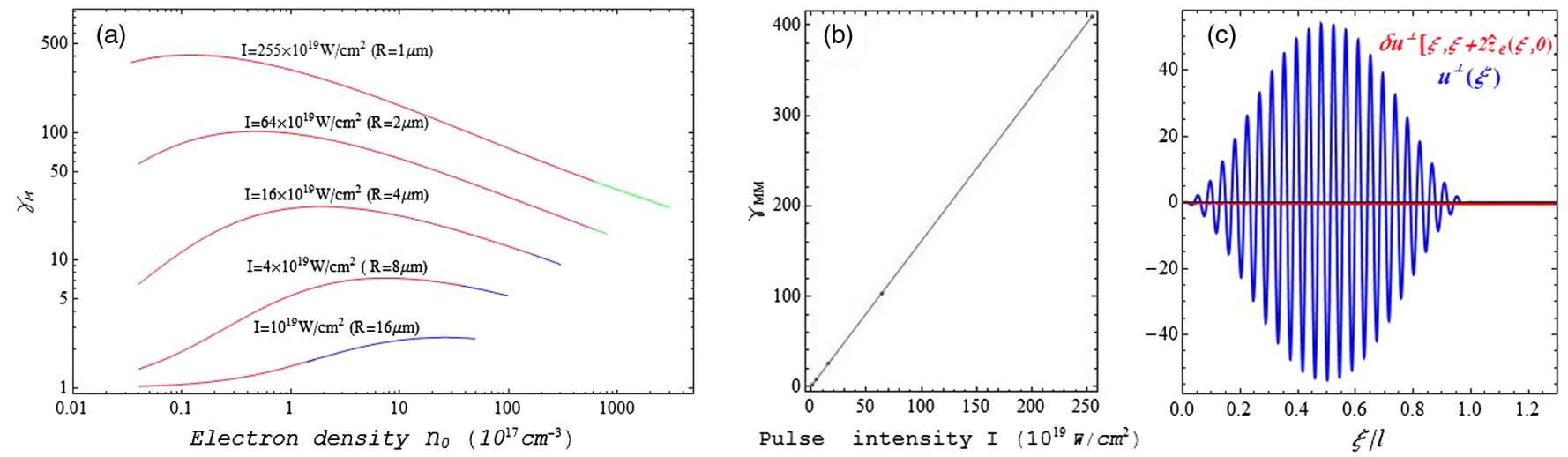

FIG. 9. (a) relativistic factor $\gamma_{M}$ of the $Z=0$ expelled electrons (the maximal one) as a function of the step-shaped initial electron density $n_{0}$, for few values of the intensity $I$; the maximum of each graph is denoted as $\gamma_{M M}$. (b): $\gamma_{M M}$ vs $I$. (c): $\boldsymbol{u}^{\perp} \&$ its correction $\delta \boldsymbol{u}^{\perp}$ along the $\boldsymbol{X}=0$ electrons' worldlines for $n_{0}=24 \times 10^{19} \mathrm{~cm}^{-3}, I=255 \times 10^{19} \mathrm{~W} / \mathrm{cm}^{2}: \delta \boldsymbol{u}^{\perp}$ is negligible. 

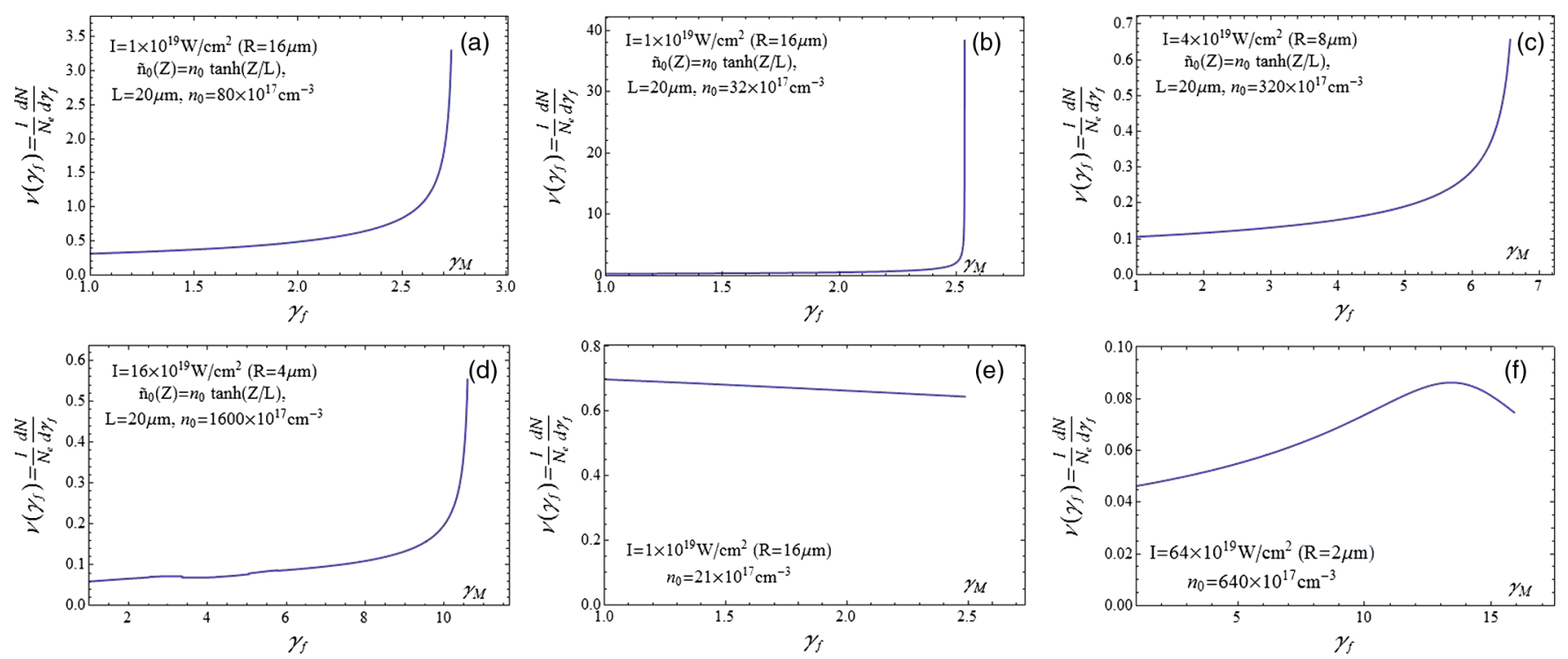

FIG. 10. Sample spectra of the expelled electrons for pulse amplitudes of the form (40) with continuous initial electron densities $\widetilde{n_{0}}(Z) \equiv n_{0} \theta(Z) \tanh (Z / L), L=20 \mu \mathrm{m}$ (graphs a-d), or step-shaped initial electron densities $\widetilde{n_{0}} \equiv n_{0} \theta(Z)$ (graphs e-f). The values of $n_{0}$ and of the average pulse intensity $I$ are the same as in Table I.

with (25), (28), (29). In Table I we report our main predictions for the same $I$ (equivalently, $R$ ) and $\widetilde{n_{0}}$. The final energies of the expelled electrons range from few to about $15 \mathrm{MeV}$. The spectra (energy distributions) are rather flat for the step-shaped densities, albeit they become more peaked near $\gamma_{M}$ as $n_{0}$ grows; if $\widetilde{n_{0}}(Z)$ grows smoothly from zero to about the asymptotic value $n_{0}$ in the interval $0 \leq Z \leq L \sim 20 \mu \mathrm{m}$, they can be made much better (almost monochromatic) by tuning $L$. The collimation of the expelled electron bunch is very good, by (33); in all cases considered in Table I we find deviations $\beta_{f}^{\perp} / \beta_{f}^{z}$ of $1 \div 2$ milliradiants for the $(\rho, Z)=(0,0)$ and $4 \div 10$ milliradiants for the $(\rho, Z)=\left(0,0.9 Z_{M}\right)$ expelled electrons.
We now discuss the conditions guaranteeing the validity of our model. The comments after (34) show for all $\xi$ the invertibility of the maps $\hat{z}_{e}(\xi, \cdot): Z \mapsto z$ in the interval $0 \leq Z \leq Z_{M}$, and therefore the self-consistency of this 2-fluid MHD model, in the step-shaped density case; numerical study of the map $\hat{z}_{e}(\cdot, \xi): Z \mapsto z$ shows that this holds true also in the continuous density case. Numerical computations show that (25) is fulfilled at least on the $Z \leq$ $Z_{M}$ electrons' worldlines, even with the highest densities considered here (see, e.g., Fig. 9 right). Finally, the data in Table I show that (28), (29) are fulfilled.

If we choose $\epsilon_{s}(\xi)$ as the cutoff Gaussian, instead of the cutoff polynomial, convergence of numerical

TABLE I. Sample inputs and outputs for possible experiments. In the "p, g" columns the initial electron densities are step-shaped, $\widetilde{n_{0}}(Z)=n_{0} \theta(Z)$, and the amplitudes are resp. of the Gaussian, polynomial forms (39), (40); in the "PoP14" columns we report results computed in [13] with poorer approximation. In the "cg,cp" columns the initial electron densities are the continuous ones $\widetilde{n_{0}}(Z)=$ $n_{0} \theta(Z) \tanh (Z / L)$ with $L=20 \mu \mathrm{m}$, and the amplitudes are resp. of the forms (39), (40).

pulse energy $\mathcal{E} \simeq 5 \mathrm{~J}$, wavelength $\lambda \simeq 0.8 \mu \mathrm{m}$, fwhm $l^{\prime} \simeq 7.5 \mu \mathrm{m}$, spot radius $R \simeq 1 \div 16 \mu \mathrm{m}$

\begin{tabular}{|c|c|c|c|c|c|c|c|c|c|c|c|c|c|c|}
\hline & $\mathrm{p}$ & PoP14 & $\mathrm{p}$ & PoP14 & $\mathrm{p}$ & g & $\mathrm{cp}$ & $\mathrm{cg}$ & $\mathrm{cp}$ & $\mathrm{cg}$ & $\mathrm{cp}$ & $\mathrm{cg}$ & $\mathrm{cp}$ & $\mathrm{cg}$ \\
\hline Pulse spot radius $R(\mu \mathrm{m})$ & 15 & 15 & 16 & 16 & 2 & 2 & 16 & 16 & 16 & 16 & 8 & 8 & 4 & 4 \\
\hline Mean intensity $I\left(10^{19} \mathrm{~W} / \mathrm{cm}^{2}\right)$ & 1.1 & 1.1 & 1 & 1 & 64 & 64 & 1 & 1 & 1 & 1 & 4 & 4 & 16 & 16 \\
\hline Initial el. density $n_{0}\left(10^{18} \mathrm{~cm}^{-3}\right)$ & .64 & .64 & .64 & .64 & 64 & 64 & 3.2 & 3.2 & 8 & 8 & 32 & 32 & 160 & 160 \\
\hline Ratio $\left[t_{\mathrm{ex}}(0)-\bar{t}(0)\right] c / R$ & 0.8 & & .75 & & 1.3 & 1.9 & 0.8 & 0.8 & 0.6 & 0.7 & 1.1 & 1.1 & 1.7 & 2.1 \\
\hline Ratio $r / R$ & 0.6 & & 0.7 & & 1 & 1 & 0.6 & 0.7 & 0.8 & 0.9 & 0.8 & 1 & 1 & 1 \\
\hline Ratio $\Delta x_{e}^{M} / R$ & .02 & & .02 & & .14 & .09 & .02 & .19 & .02 & .17 & .05 & .04 & .1 & .06 \\
\hline Maximal relativistic factor $\gamma_{M}$ & 2.5 & 1.83 & 2.3 & 1.65 & 16 & 14 & 2.5 & 2.4 & 2.7 & 2.6 & 6.6 & 5.6 & 11 & 8.1 \\
\hline Max. expulsion energy $H(\mathrm{MeV})$ & 1.3 & 0.94 & 1.2 & 0.9 & 8.1 & 7.2 & 1.3 & 1.2 & 1.4 & 1.3 & 3.4 & 2.9 & 5.4 & 4.2 \\
\hline Tot expelled charge $|Q|\left(10^{-10} \mathrm{C}\right)$ & 1.7 & 3.8 & 2.2 & 3.2 & 3.7 & 3.1 & 1.9 & 2.2 & 3.7 & 2.9 & 3.5 & 4 & 3.8 & 3.5 \\
\hline Tot. exp. kin. energy $\mathrm{E}\left(10^{-4} \mathrm{~J}\right)$ & 0.7 & & 0.7 & & 16 & 12 & 0.8 & 0.8 & 1.6 & 1.1 & 4.5 & 4.0 & 8.6 & 5.8 \\
\hline
\end{tabular}


computations is slower, but the outcomes do not differ significantly. Sample computations show that choices of other continuous $\widetilde{n_{0}}(Z)$ lead to similar results, provided the function $\widetilde{n_{0}}(Z)$ is increasing and significantly approaches the asymptotic value $n_{0}$ in the interval $0 \leq Z \leq L \sim 20 \mu \mathrm{m}$.

\section{DISCUSSION, FINAL REMARKS, CONCLUSIONS}

These results show that indeed the slingshot effect is a promising acceleration mechanism of electrons, in that it extracts from the targets highly collimated bunches of electrons with spectra which can be made peaked around the maximum energies by adjusting $R, \widetilde{n_{0}}$; with laser pulses of a few joules and duration of few tens of femtoseconds (as available today in many laboratories) we find that the latter range up to about ten $\mathrm{MeV}$ (it would increase with more energetic pulses). The spectra (distributions of electrons as functions of the final relativistic factor $\gamma_{f}$ ), their dependence on the electron density and pulse intensity, the collimation and the backward direction of expulsion in principle allow to discriminate the slingshot effect from the LWFA or other acceleration mechanisms. In Table I and Fig. 10 we have reported detailed quantitative predictions of the main features of the effect for some possible choices of parameters in experiments at the present FLAME, the future upgraded ILIL facilities, or similar laboratories. Low density gases or aerogels (the lightest solids available today) are targets with appropriate electron densities.

The steepest $z$-oriented density gradient of a gas sample isolated in vacuum is attained just outside a nozzle expelling a supersonic gas jet in the $x y$ plane; across the lateral border of the jet the density may vary from about zero to almost the asymptotic value $n_{0}$ in about $L \sim 20 \mu \mathrm{m}$ [27]. Hence if we choose a supersonic helium jet as the laser pulse target the initial electron density is reasonably approximated by the choice $\widetilde{n_{0}}(Z)=n_{0} \theta(Z) \tanh (Z / L)$, and the predictions of Table I, Fig. 10(a-d) are reliable. By the way, the values of $n_{0}$ considered in Table $\mathrm{I}$ are considerably higher than in typical LWFA experiments.

Step-shaped $\widetilde{n_{0}}(Z)$ are unrealistic approximations of densities of gas samples, but reasonable ones of solids (for which $\Delta Z \ll \lambda$ ), provided $n_{0}$ exceeds $48 \times 10^{18} \mathrm{~cm}^{-3}$, which is the electron density of aerographene (the lightest aerogel so far: mass density $=0.00016 \mathrm{~g} / \mathrm{cm}^{3}$ ). Silica areogels, with a wide range of densities from 0.7 to $0.001 \mathrm{~g} / \mathrm{cm}^{3}$, electron densities of the order of $10^{20} / \mathrm{cm}^{-3}$ and porosity from $50 \mathrm{~nm}$ down to $2 \mathrm{~nm}$ in diameter (i.e. much smaller than $\lambda$ ) have been produced and extensively studied [30,31]. Therefore the results of the last two "p,g" columns of Table I [and the corresponding spectra, Fig. 10(e)] are applicable to aerogels, while those of the first four are presently only of academic interest.

The quantitative predictions of our model are based on a rather rigorous plane-wave, 2-fluid magnetohydrodynamic model [32] and simple, but heuristic approximations for the 3D corrections, which certainly affect their liability. We welcome numerical 3D simulations (particle-in-cell ones, etc.) to improve the latter. Experimental tests are easily feasible with the equipments presently available in many laboratories. We welcome experiments testing the effect.

\section{ACKNOWLEDGMENTS}

We are pleased to thank R. Fedele, L. Gizzi for stimulating discussions. G. F. acknowledges partial support by UniNA \& Compagnia di San Paolo under the grant STAR Program 2013, and by COST Action MP1405 Quantum Structure of Spacetime.

\section{APPENDIX A: FINITE $\boldsymbol{R}$ CONDITIONS}

As known, for any spacetime region $\mathcal{D}$ its future Cauchy development $D^{+}(\mathcal{D})$ is defined as the set of all points $x$ for which every past-directed causal (i.e. non-spacelike) line through $x$ intersects $\mathcal{D}$ (see Fig. 11 left). Causality implies:
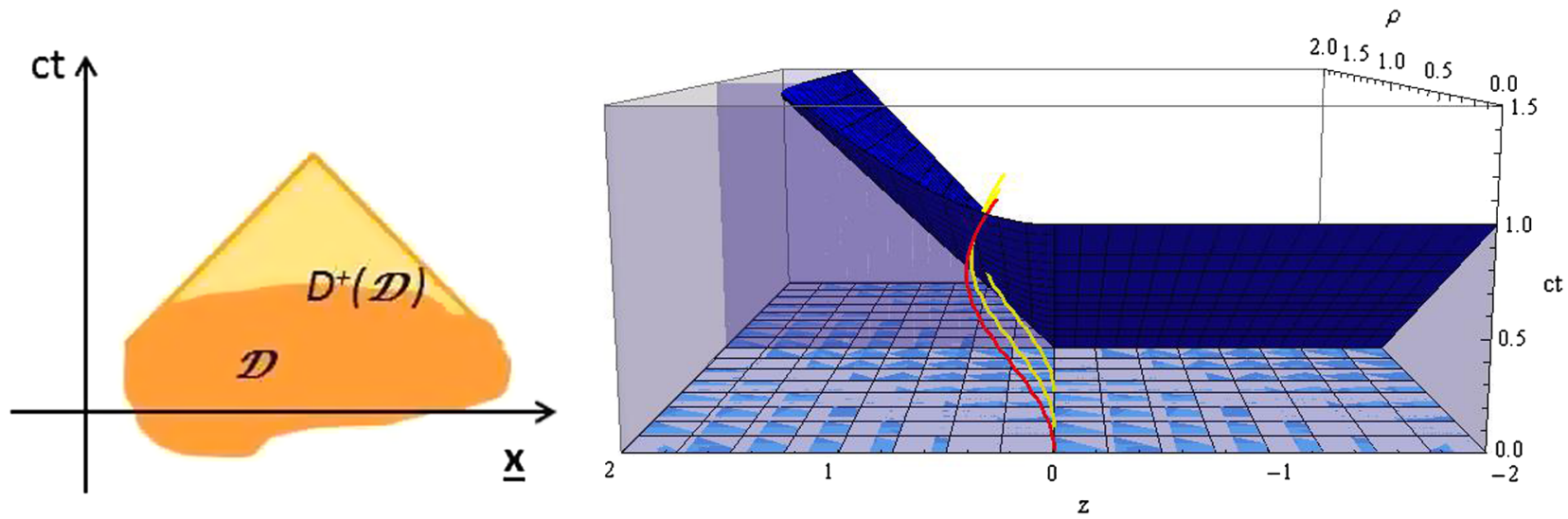

FIG. 11. Left: future Cauchy development $D^{+}(\mathcal{D})$ of a generic domain $\mathcal{D}$. Right: $\mathcal{D}_{1}^{0}$ (light blue) and $D^{+}\left(\mathcal{D}_{1}^{0}\right)($ shaded region between the blue and light blue hypersurfaces) in $(\rho, z, c t)$ coordinates (we have dropped the inessential angle $\varphi$ ); worldlines of the $\boldsymbol{X}=0$ electrons (red) and of a couple of off- $\vec{z}$-axis electrons (yellow); the former remain in $D^{+}\left(\mathcal{D}_{1}^{0}\right)$ longer. 
If two solutions of the system of dynamic equations coincide in some open spacetime region $\mathcal{D}$, then they coincide also in $D^{+}(\mathcal{D})$. Therefore, knowledge of one solution determines also the other (which we will distinguish by adding a prime to all fields) in $D^{+}(\mathcal{D})$.

In the problem at hand the solutions are exactly known for $t \leq 0$, i.e. before the laser-plasma interaction begins. We use causality adopting: (1) as $\mathcal{D}$ a region $\mathcal{D}_{R}^{0}$ (see Fig. 11 right) of equations $-\epsilon \leq t \leq 0$ and either $\rho<R$ or $z>0$, with some $\epsilon>0$ (we can take also $\epsilon=0$ if we assign on $\mathcal{D}_{R}^{0}$ also the time derivatives of the $A^{\mu}, \boldsymbol{u}$ ); (2) as the known solution the plane one induced (Sec. II) by the plane transverse electromagnetic potential, which can be approximated as $\boldsymbol{A}^{\perp}(t, z)=\boldsymbol{\alpha}^{\perp}(c t-z)$ under the assumption (25); (3) as the unknown solution the "real" one induced by the "real" laser pulse $A_{f}^{\mu}(t, \boldsymbol{x})$, which we approximate as a potential leading to Eq. (3). It is easy to show that $D^{+}\left(\mathcal{D}_{R}^{0}\right)$ is the union of three regions, resp. of equations: (a) $z \geq c t \geq 0$; (b) $c t \geq z \geq 0$ and $\rho+\sqrt{c^{2} t^{2}-z^{2}} \leq R$; (c) $t \geq 0, z<0$ and $\rho+c t \leq R$ (see Fig. 11). In $D^{+}\left(\mathcal{D}_{R}^{0}\right)$ the two solutions coincide, in particular a "real" electron worldline $\boldsymbol{x}_{e}{ }^{\prime}(t, \boldsymbol{X})$ remains equal to the plane solution worldline $\boldsymbol{x}_{e}(t, \boldsymbol{X})$ as long as $\boldsymbol{x}_{e}(t, \boldsymbol{X}) \in D^{+}\left(\mathcal{D}_{R}^{0}\right)$.

By continuity, we expect that the two solutions remain close to each other also in a neighborhood of $D^{+}\left(\mathcal{D}_{R}^{0}\right)$. This is confirmed by estimates [13] involving the retarded electromagnetic potential (in the Lorentz gauge $\partial \cdot A=0$ )

$$
A^{\mu}(t, \boldsymbol{x})=A_{f}^{\mu}(t, \boldsymbol{x})+\int d^{3} x^{\prime} \frac{j^{\mu}\left[t_{r}\left(t, \boldsymbol{x}-\boldsymbol{x}^{\prime}\right), \boldsymbol{x}^{\prime}\right]}{\left|\boldsymbol{x}-\boldsymbol{x}^{\prime}\right|},
$$

i.e. the general solution of the Maxwell equation $\square A^{\mu}=$ $4 \pi j^{\mu}$ with a current $j^{\mu}(t, \boldsymbol{x})$ vanishing for $t<0$; here $t_{r}\left(t, \boldsymbol{x}-\boldsymbol{x}^{\prime}\right) \equiv t-\left|\boldsymbol{x}-\boldsymbol{x}^{\prime}\right| / c, \quad A_{f}^{\mu}(t, \boldsymbol{x}) \quad$ fulfills $\square A_{f}^{\mu}=0$ (determining the $t \rightarrow-\infty$ behavior), and $\boldsymbol{E}=\frac{-1}{c} \partial_{t} \boldsymbol{A}-\nabla A^{0}$, $\boldsymbol{B}=\nabla \times \boldsymbol{A}$. Since the formation of $C_{R}^{\zeta}$ is completed at $t=\bar{t}(0)$, and the "information" [encoded in (A1)] about the finite radius of $C_{R}^{\zeta}$ takes a time $R / c$ to go from the lateral surface $\rho=R$ to the $\vec{z}$-axis, then if Eq. (28) is fulfilled the $\boldsymbol{X}=0$ electrons (red worldline in Fig. 11) move approximately as in Sec. II until the expulsion. Similarly, the $Z \simeq 0, \rho \lesssim r$ electrons (yellow worldlines in Fig. 11) move approximately as in Sec. II until $\bar{t}+(R-r) / c$, i.e. get the main backward boost (acceleration is maximal around $\bar{t}$ ). Equation $(28)_{2}$ is equivalent to

$$
\begin{aligned}
& t_{\mathrm{ex}} \lesssim l / c ; \Rightarrow r \simeq R \\
& \text { or } 0<\left(t_{\mathrm{ex}}-l / c\right) v_{a}^{\rho}<R \Rightarrow r \simeq R-\left(t_{\mathrm{ex}}-l / c\right) v_{a}^{\rho}>0 \text {. }
\end{aligned}
$$

If the left-hand side of the first line is fulfilled the surface electrons are expelled while the laser pulse is still entering the bulk and thus producing an outward force that keeps the LE out of $C_{R}^{\zeta}$. Otherwise, the left-hand side of the second line ensures that the distance inward traveled by the most dangerous LE (the $Z=0$ ones) after the pulse has completely entered the bulk is less than $R$; $v_{a}^{\rho}$ stands for the average $\rho$-component of the velocity of these LE. By geometric reasons $v_{a}^{\rho}<v_{a}^{z} \equiv$ average $z$-component of the $\boldsymbol{X}=0$ electrons velocity in their backward trip within the bulk; our rough estimate $v_{a}^{\rho} \simeq v_{a}^{z} / 2=\zeta /\left(t_{\mathrm{ex}}-\bar{t}\right) 2$ gives $(28)_{2}$. Equation (28) is thus explained.

\section{APPENDIX B: FINITE $R$ ENERGIES}

Using cylindrical coordinates $(y, \rho, \varphi)$ for $\boldsymbol{X}^{\prime}$, one easily finds that for $z_{e} \equiv Z+\Delta \leq 0$ the electric force generated by the static charge distribution of Fig. 5(b), the associated potential energy $m c^{2} \mathcal{U}_{r}$ and the left asymptotes of $\mathcal{U}_{r}$ are

$$
\begin{gathered}
F_{e r}^{z}(\Delta, Z) \equiv \int_{0}^{Z_{2}(Z)} d y \widetilde{n_{0}}(y) \int_{0}^{r} d \rho \frac{2 \pi e^{2} \rho\left(y-z_{e}\right)}{\left[\rho^{2}+\left(y-z_{e}\right)^{2}\right]^{3 / 2}} \\
=-2 \pi e^{2} \int_{0}^{Z_{2}(Z)} d y \frac{\widetilde{n_{0}}(y)\left(y-z_{e}\right)}{\sqrt{\left(y-z_{e}\right)^{2}+r^{2}}}+4 \pi e^{2} \tilde{N}(Z) . \\
\mathcal{U}_{r}(\Delta, Z) \equiv \frac{\mu}{2} \int_{0}^{Z_{2}(Z)} d y \tilde{n_{0}}(y)\left[\sqrt{y^{2}+r^{2}}-\sqrt{(y-Z-\Delta)^{2}+r^{2}}\right]-\mu \tilde{N}(Z) \Delta-\mu \tilde{\mathcal{N}}(Z), \\
\mathcal{U}_{r}(-\infty, Z)=\frac{\mu}{2} \int_{0}^{Z_{2}(Z)} d y \tilde{n_{0}}(y)\left[\sqrt{y^{2}+r^{2}}-y\right]+\mu \int_{0}^{Z} d y \tilde{n_{0}}(y) y .
\end{gathered}
$$

Here $\mu \equiv 4 \pi e^{2} / m c^{2} . \mathcal{U}_{r}$ is continuous in $(-Z, Z)$, since we have chosen $\mathcal{U}(-Z, Z)$ as the ( $\Delta$-independent) "additive constants." Energy conservation implies

$$
\gamma+\mathcal{U}_{r}(\Delta, Z)=\gamma[\hat{l, Z}]+\mathcal{U}_{r}[\hat{\Delta}(l, Z), Z]=\hat{\gamma}\left[\xi_{\mathrm{ex}}(Z), Z\right]+\mathcal{U}_{r}(-Z, Z) .
$$


The last equality holds only if $\hat{z}_{e}(l, Z) \geq 0$, i.e. $l \leq \xi_{\mathrm{ex}}(Z)$; the right-hand side is the electrons' energy when expelled from the bulk. This leads to the final relativistic factor

$$
\begin{aligned}
\gamma_{f}(Z) & =\hat{\gamma}(l, Z)-\mu \tilde{N}(Z) \hat{z}_{e}(l, Z)+\frac{\mu}{2} \int_{0}^{Z_{2}(Z)} d y \tilde{n_{0}}(y)\left[y-\sqrt{\left[y-\hat{z}_{e}(l, Z)\right]^{2}+r^{2}}\right] \\
& =\hat{\gamma}\left[\xi_{\mathrm{ex}}(Z), Z\right]+\frac{\mu}{2} \int_{0}^{Z_{2}(Z)} d y \tilde{n_{0}}(y)\left[y-\sqrt{y^{2}+r^{2}}\right] \quad \text { if } l \leq \xi_{\mathrm{ex}}(Z) .
\end{aligned}
$$

Deriving this and the identity $\tilde{N}\left[Z_{2}(Z)\right]=2 \tilde{N}(Z)$ we find $\widetilde{n_{0}}\left[Z_{2}(Z)\right] \frac{d Z_{2}}{d Z}=2 \widetilde{n_{0}}(Z)$ and that, as claimed, $\gamma_{f}(Z)$ is strictly decreasing, since $d \gamma_{f} / d Z$ is negative-definite:

$$
\begin{aligned}
\frac{d \gamma_{f}}{d Z}= & \frac{\partial \hat{\gamma}(l, Z)}{\partial Z}-\frac{\mu}{2} \frac{d Z_{2}}{d Z} \tilde{n}_{0}\left[Z_{2}(Z)\right]\left[\sqrt{\left[Z_{2}(Z)-\hat{z}_{e}(l, Z)\right]^{2}+r^{2}}-Z_{2}(Z)\right] \\
& -\mu \tilde{n_{0}}(Z) \hat{z}_{e}(l, Z)-\mu \tilde{N}(Z) \frac{\partial \hat{z}_{e}(l, Z)}{\partial Z}=\frac{\partial \hat{\gamma}}{\partial Z}(l, Z)-\mu \tilde{N}(Z) \frac{\partial \hat{z}_{e}}{\partial Z}(l, Z) \\
& -\mu \tilde{n_{0}}(Z)\left[\sqrt{\left[Z_{2}(Z)-\hat{z}_{e}(l, Z)\right]^{2}+r^{2}}-Z_{2}(Z)+\hat{z}_{e}(l, Z)\right] .
\end{aligned}
$$

For the step-shaped initial density, setting $\phi \equiv \hat{\Delta}(l, Z)-Z$,

$$
\begin{gathered}
\mathcal{U}_{r}(\Delta, Z)=\frac{M}{4}\left[(\Delta-Z) \sqrt{(\Delta-Z)^{2}+r^{2}}-4 Z(\Delta+Z)+r^{2} \sinh ^{-1} \frac{\Delta-Z}{r}-(\Delta+Z) \sqrt{(\Delta+Z)^{2}+r^{2}}-r^{2} \sinh ^{-1} \frac{\Delta+Z}{r}\right. \\
\left.+2 Z^{2}+2 Z \sqrt{4 Z^{2}+r^{2}}+r^{2} \sinh ^{-1} \frac{2 Z}{r}\right] \\
\mathcal{U}_{r}(-\infty, Z)=\frac{M Z}{2}\left[\sqrt{4 Z^{2}+r^{2}}-Z+\frac{r^{2}}{2 Z} \sinh ^{-1} \frac{2 Z}{R}\right], \\
\gamma_{f}(Z)=\hat{\gamma}(l, Z)+\frac{M}{4}\left\{\phi \sqrt{\phi+r^{2}}-4 Z \hat{\Delta}(l, Z)+r^{2} \sinh ^{-1} \frac{\phi}{r}-[\phi+2 Z] \sqrt{[\phi+2 Z]^{2}+r^{2}}-r^{2} \sinh ^{-1} \frac{\phi+2 Z}{r}\right\} \\
=\hat{\gamma}\left[\xi_{\mathrm{ex}}(Z)\right]+M\left[Z^{2}-\frac{Z}{2} \sqrt{4 Z^{2}+r^{2}}-\frac{r^{2}}{4} \sinh ^{-1} \frac{2 Z}{r}\right] \quad \text { if } l \leq \xi_{\mathrm{ex}}(Z) .
\end{gathered}
$$

If $l \leq \xi_{\text {ex }}(Z)$ then $\partial_{Z} \hat{\gamma}=0=\partial_{Z} \hat{\Delta}$ at $\xi=\xi_{\text {ex }}(Z)$, eq. (B5) reduces to $d \gamma_{f} / d Z=M\left[Z-\sqrt{4 Z^{2}+r^{2}}\right]$, and (32) to

$$
1 / \nu(\gamma)=M Z_{M}\left[\sqrt{4 Z^{2}+r^{2}}-Z\right]_{Z=\hat{Z}(\gamma)} .
$$

[1] T. Tajima and J. M. Dawson, Laser Electron Accelerator, Phys. Rev. Lett. 43, 267 (1979).

[2] D. Strickland and G. Mourou, Compression of amplified chirped optical pulses, Opt. Commun. 56, 219 (1985).

[3] M. D. Perry and G. Mourou, Terawatt to petawatt subpicosecond lasers, Science 264, 917 (1994); and references therein.

[4] L. M. Gorbunov and V. I. Kirsanov, ZhETF 93, 509 (1987) [Excitation of plasma waves by an electromagnetic wave packet, Sov. Phys. JETP 66, 290 (1987)].

[5] P. Sprangle, E. Esarey, A. Ting, and G. Joyce, Laser wakefield acceleration and relativistic optical guiding, Appl. Phys. Lett. 53, 2146 (1988).
[6] S. P. Mangles, C. D. Murphy, Z. Najmudin, A. G. Thomas, J. L. Collier, A. E. Dangor, E. J. Divall, P. S. Foster, J. G. Gallacher, C. J. Hooker, D. A. Jaroszynski, A. J. Langley, W. B. Mori, P. A. Norreys, F. S. Tsung, R. Viskup, B. R. Walton, and K. Krushelnick, Monoenergetic beams of relativistic electrons from intense laser-plasma interactions, Nature (London) 431, 535 (2004).

[7] C. G. R. Geddes, Cs. Tóth, J. van Tilborg, E. Esarey, C. B. Schroeder, D. Bruhwiler, C. Nieter, J. Cary, and W. P. Leemans, High-quality electron beams from a laser wakefield accelerator using plasma-channel guiding, Nature (London) 431, 538 (2004). 
[8] J. Faure, Y. Glinec, A. Pukhov, S. Kiselev, S. Gordienko, E. Lefebvre, J.-P. Rousseau, F. Burgy, and V. Malka, A laserplasma accelerator producing monoenergetic electron beams, Nature (London) 431, 541 (2004).

[9] X. Wang et al., Quasi-monoenergetic laser-plasma acceleration of electrons to $2 \mathrm{GeV}$, Nat. Commun. 4, 1988 (2013).

[10] W. P. Leemans, A. J. Gonsalves, H.-S. Mao, K. Nakamura, C. Benedetti, C. B. Schroeder, Cs. Tóth, J. Daniels, D. E. Mittelberger, S. S. Bulanov, J.-L. Vay, C. G. R. Geddes, and E. Esarey, Multi-GeV Electron Beams from CapillaryDischarge-Guided Subpetawatt Laser Pulses in the Self-Trapping Regime, Phys. Rev. Lett. 113, 245002 (2014).

[11] A. Irman, M. J. H. Luttikhof, A. G. Khachatryan, F. A. van Goor, J. W. J. Verschuur, H. M. J. Bastiaens, and K.-J. Boller, Design and simulation of laser wakefield acceleration with external electron bunch injection in front of the laser pulse, J. Appl. Phys. 102, 024513 (2007).

[12] C. Joshi, Plasma accelerators, Sci. Am. 294, 40 (2006).

[13] G. Fiore, R. Fedele, and U. de Angelis, The slingshot effect: A possible new laser-driven high energy acceleration mechanism for electrons, Phys. Plasmas 21, 113105 (2014).

[14] $T_{H}$ grows with the oscillation amplitude $\zeta$, but goes to the nonrelativistic period $T_{H}^{n r}=\sqrt{\pi m / n_{0} e^{2}}$ as $\zeta \rightarrow 0$.

[15] If $\tau \ll T_{H}$, then while the pulse is passing $F_{e}^{z}$ can be neglected, and the motion of the electron is close to the one in vacuum; the backward acceleration takes place afterwards and is due only to $F_{e}^{z}$, hence the final energy is smaller. Whereas if $\tau \gg T_{H}$-which was the standard situation in laboratories until a couple of decades agothen $F_{p} v^{z}$ oscillates many times about $0, W \simeq 0$, and the backward acceleration is washed out.

[16] G. Fiore, On plane-wave relativistic electrodynamics in plasmas and in vacuum, J. Phys. A 47, 225501 (2014).

[17] G. Fiore and S. De Nicola, A "slingshot" laser-driven acceleration mechanism of plasma electrons, Nucl. Instrum. Methods Phys. Res., Sect. A 829, 104 (2016).

[18] G. Fiore, On very short and intense laser-plasma interactions, Ricerche Mat. (in press), doi: 10.1007/s11587016-0270-3.

[19] A. A. Sahai, F. S. Tsung, A. R. Tableman, W. B. Mori, and T.C. Katsouleas, Relativistically induced transparency acceleration of light ions by an ultrashort laser pulse interacting with a heavy-ion-plasma density gradient, Phys. Rev. E 88, 043105 (2013).

[20] A. A. Sahai, Motion of the plasma critical layer during relativistic-electron laser interaction with immobile and comoving ion plasma for ion acceleration, Phys. Plasmas 21, 056707 (2014).

[21] J. Badziak, S. Glowacz, S. Jablonski, P. Parys, J. Wolowski, H. Hora, J. Krása, L. Láska, and K. Rohlena, Production of ultrahigh ion current densities at skin-layer subrelativistic laser-plasma interaction, Plasma Phys. Controlled Fusion 46, B541 (2004).

[22] Albeit the pump (3) violates the Maxwell equations (due to the $\rho$-dependence), we adopt it as for our purposes it is essentially equivalent to one that fulfills the Maxwell equations and at the time of impact coincides with it in the main part of its support, while rapidly decaying outside (this and similar approximations, e.g. the paraxial one, are currently used in the literature).

[23] G. Fiore, Travelling waves and a fruitful 'time' reparametrization in relativistic electrodynamics, arXiv:1607.03482.

[24] G. Fiore, On plane waves in diluted relativistic cold plasmas, Acta Appl. Math. 132, 261 (2014).

[25] G. Fiore, A plane-wave model of the impact of short laser pulses on plasmas (to be published).

[26] $\hat{u}^{z}, \hat{\gamma}, \hat{\boldsymbol{Y}}, \hat{\Xi}, \hat{\boldsymbol{x}}_{e}$ reduce to the $u_{e}^{z(0)}, \gamma_{e}^{(0)}, \boldsymbol{Y}_{e}, \Xi_{e}, \boldsymbol{x}_{e}^{(0)}$ of [16] if $\widetilde{n_{0}}(Z) \equiv 0$.

[27] L. A. Gizzi et al., Laser-plasma acceleration with FLAME and ILIL ultraintense lasers, Appl. Sci. 3, 559 (2013).

[28] L. Gizzi (private communication).

[29] We thank L. Gizzi for this remark.

[30] A.C. Pierre and G. M. Pajonk, Aerogels and their applications, Chem. Rev. 102, 4243 (2002).

[31] A. P. Ambekar and P. Bagade, A review on: AerogelWorld's lightest solid, Popular Plastics \& Packaging 51, 96 (2006).

[32] There is no need of a recourse to kinetic theory taking collisions into account, e.g., by BGK [33] equations or effective linear inheritance relations [34].

[33] P. L. Bhatnagar, E. P. Gross, and M. Krook, A model for collision processes in gases. I. Small amplitude processes in charged and neutral one-component systems, Phys. Rev. 94, 511 (1954).

[34] G. Fiore, A. Maio, and P. Renno, On the initial-value problem in a cold plasma model, Ric. Mat. 63, 157 (2014). 